ARTICLE

https://doi.org/10.1038/s41467-019-09878-4

\title{
Collagen-rich airway smooth muscle cells are a metastatic niche for tumor colonization in the lung
}

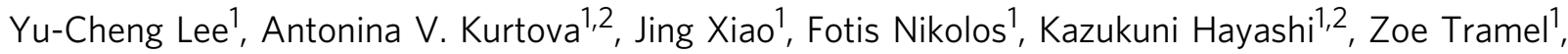
Antrix Jain (1) ${ }^{3}$, Fengju Chen ${ }^{4}$, Mithil Chokshi ${ }^{5}$, Ciaran Lee (iD ${ }^{5}$, Gang Bao ${ }^{5}$, Xiang Zhang ${ }^{1}$, Jianjun Shen ${ }^{6}$, Qianxing Mo ${ }^{7}$, Sung Yun Jung $\mathbb{D}^{3}{ }^{3}$, David Rowley ${ }^{1} \&$ Keith Syson Chan ${ }^{1,4}$

Metastases account for the majority of cancer deaths. While certain steps of the metastatic cascade are well characterized, identification of targets to block this process remains a challenge. Host factors determining metastatic colonization to secondary organs are particularly important for exploration, as those might be shared among different cancer types. Here, we showed that bladder tumor cells expressing the collagen receptor, CD167a, responded to collagen I stimulation at the primary tumor to promote local invasion and utilized the same receptor to preferentially colonize at airway smooth muscle cells (ASMCs) - a rich source of collagen III in lung. Morphologically, COL3-CD167a-driven metastatic foci are uniquely distinct from typical lung alveolar metastatic lesions and exhibited activation of the CD167a-HSP90-Stat3 axis. Importantly, metastatic lung colonization could be abrogated using an investigational drug that attenuates Stat3 activity, implicating this seed-and-soil interaction as a therapeutic target for eliminating lung metastasis.

\footnotetext{
${ }^{1}$ Department of Molecular and Cellular Biology, Baylor College of Medicine, Houston, TX 77030, USA. ${ }^{2}$ Graduate Program in Translational Biology and Molecular Medicine, Baylor College of Medicine, Houston, TX 77030, USA. ${ }^{3}$ Department of Biochemistry and Molecular Biology, Mass Spectrometry Proteomics Core, Baylor College of Medicine, Houston, TX 77030, USA. ${ }^{4}$ Dan L Duncan Cancer Center, Baylor College of Medicine, Houston, TX 77030, USA. ${ }^{5}$ Department of Bioengineering, Rice University Houston, Houston, TX 77030, USA. ${ }^{6}$ University of Texas M.D. Anderson Cancer Center, Houston, TX 77030, USA. ${ }^{7}$ Department of Biostatistics \& Bioinformatics, H. Lee Moffitt Cancer Center \& Research Institute, Tampa, FL 33612, USA. Correspondence and requests for materials should be addressed to K.S.C. (email: keithsyson2819@gmail.com)
} 
M etastasis is a multi-step process that includes, but is not limited to local invasion, intravasation of tumor cells into the circulation and extravasation of circulating tumor cells (CTCs) from the circulation into distal organs for metastatic colonization ${ }^{1-6}$. The 5-year survival rate of patients diagnosed with metastatic bladder urothelial carcinoma is $\sim 6 \%$, posing a major clinical challenge ${ }^{7-10}$. Previous studies in human bladder urothelial carcinomas have elegantly investigated the mechanistic contribution of tumor cell intrinsic properties that promote metastasis ${ }^{11-13}$. However, the contribution of tumor microenvironments at both the primary tumor and metastatic sites to the metastatic process is not as well characterized ${ }^{14}$.

Historically, the extracellular matrix (ECM) was considered a passive scaffold, enclosing neighboring cancer cells and other cell types to support tissue architecture. Intriguingly, recent studies revealed the emerging roles of ECMs as a dynamic component of the tumor microenvironment, which crosstalks with cancer cells at the primary tumor site ${ }^{15-17}$. Collagens are major components of the ECM in various organs ${ }^{15}$, including the bladder. Albeit the interaction of collagens with its receptors to facilitate the metastatic cascade, has not been sufficiently explored in bladder carcinomas. Extensive studies have investigated how primary tumors create a favorable microenvironment at secondary organs-designated as the pre-metastatic niche ${ }^{18}$. However, the pre-existence of a physical metastatic niche within secondary organs (e.g. lung) to support Paget's original seed and soil hypothesis for metastatic seeding and colonization currently lacks evidential substantiality. Here, we report a collagen receptor (CD167a or Discoidin domain receptor 1) on tumor cells, which crosstalk with collagens at both the primary tumor microenvironment and a newly identified metastatic niche in the lung.

\section{Results}

Collagen I positively correlates with bladder tumor staging. Clinically, tumor (T) staging defines the invasiveness of human bladder urothelial carcinoma cells into smooth muscle and perivesical tissues pathologically ${ }^{19}$, with $\mathrm{Ta} / \mathrm{T} 1$ defining nonmuscle invasive bladder carcinomas (NMIBCs), and T2-T4 representing muscle-invasive bladder carcinomas (MIBCs). To explore a clinical connection between collagen I (COL1) and tumor cell invasion, we evaluated collagen I gene expression (COL1A1 and COL1A2) in correlation to tumor staging in three independent cohorts of bladder cancer patients [kim, $n=165^{20}$, Riester, $n=93^{21}$ and The Cancer Genome Atlas (TCGA), $n=$ $376^{22}$ ] (Fig. 1a, b). Initial analysis of COL1A1 and COL1A2 genes revealed significantly higher expression in MIBCs $(>\mathrm{T} 2)$, when compared to NMIBCs (Ta/T1) [Fig. 1a, cohort I: COL1A1 $p=1 \mathrm{E}-$ 10; COL1A2 $p=4 \mathrm{E}-5$; cohort II: COL1A1 $p=0.0018$; COL1A2 $p=0.00016$; cohort III: COL1A1 $p=0.00425$; COL1A2 $\mathrm{p}=$ $0.00385]$. Subsequent detailed analysis also revealed a significant and positive correlation between COL1A1 and COL1A2 gene expression with increasing tumor staging [Fig. 1b, cohort I: COL1A $1 p=1 \mathrm{E}-9$ and COL1A2 $p=7 \mathrm{E}-4, \mathrm{Ta} / \mathrm{T} 1(n=103)$, T2 $(n=26)$, T3 $(n=13), \mathrm{T} 4(n=23)$; cohort II: COL1A1 $p=0.0036$ and COL1A2 $p=4 \mathrm{E}-04, \mathrm{Ta} / \mathrm{T} 1(n=15), \mathrm{T} 2(n=17), \mathrm{T} 3(n=42)$, T4 $(\mathrm{n}=19)$; cohort III: COL1A1 $p=3.32 \mathrm{E}-11$, and COL1A2 $p=1.61 \mathrm{E}-09, \mathrm{Ta} / \mathrm{T} 1 \quad(n=4), \mathrm{T} 2 \quad(n=120), \mathrm{T} 3 \quad(n=194), \mathrm{T} 4$ $(n=58)]$. Ta/T1 tumors are papillary and non-muscle invasive carcinomas, respectively. T2 tumors represent carcinomas beginning to invade into the smooth muscles, T3 tumors represent those that have invaded through muscularis into perivesical adipose tissues, and T4 tumors have spread beyond perivascular adipose tissues into nearby organs. Since collagens constitute the major proteins comprising the ECM, and were also recognized as functional ligands that facilitate crosstalk with neighboring cancer cells ${ }^{15-17,23}$, the findings in Fig. 1a, b implicated the clinical relevance to further investigate collagen I and its crosstalk with collagen receptor on cancer cells to regulate tumor invasion.

Collagen I enhances tumor cell migration and invasion. To evaluate COL1 as a functional ligand to modulate the invasive properties of human bladder cancer cells, we utilized a weakly metastatic cancer cell line T2 $24^{24}$, as well as primary cultures from a metastatic patient-derived xenograft (Human PDX) to assess their responses to exogenous COL1 treatment in vitro. First, to examine whether COL1 regulates cell migration, cancer cells were subjected to an in vitro scratch assay ${ }^{25}$, in the absence or presence of increasing exogenous $\operatorname{COL} 1\left(25,50\right.$, and $\left.100 \mu \mathrm{g} \mathrm{ml}^{-1}\right)$ (Fig. 1c, d). COL1 treatment consistently enhanced wound closure in a dose-dependent manner, in both T24 and human PDX models, when compared to vehicle control (Fig. 1c, d); thus, confirming COL1 is not merely a passive ECM, but also a functional ligand that promotes cell migration.

Next, to explore the functional impact of COL1 in modulating cancer cell invasion through ECM, we employed various 3dimensional (3D) models to better recapitulate in vivo conditions. When cancer cells were cultured at low density within a thick layer of matrigel matrix (Fig. 1e, upper panels) or collagen-matrigel mixed matrix (Fig. 1e, lower panels), they exhibited significantly distinct cellular morphologies. Cancer cells embedded within the collagen-matrigel matrix exhibited extensive sprouting structures with a higher propensity to invade through the COL1-enriched matrix (Fig. 1e, lower panels), while cells embedded within matrigel remained primarily spherical (Fig. 1e, upper panels). Collectively, extracellular COL1 consistently increases cell migration in 2D; however, its functional impact on cancer cells is much more pronounced within a $3 \mathrm{D}$ matrix. To quantify the capacity of COL1 to stimulate invasion through $3 \mathrm{D}$ matrice, we employed another model comprising two layers of matrices ${ }^{26}$ (Fig. 1f, g). This 3D invasion assay revealed that COL1 was sufficient to stimulate a longer invading distance by cancer cells into the bottom matrix (Fig. 1f), as well as increased the total number of cells that invaded into the matrix in a dose-dependent manner (Fig. 1g). Collectively, these results convincingly demonstrated collagen I is not only a passive ECM component, but also a functional ligand that promoted cancer cell sprouting and invasion through ECM.

CD167a as a collagen receptor in human MIBCs. To identify the relevant collagen receptor, we analyzed gene expression dataset of human MIBCs and identified a patient population coexpressing both COL1A1/1A2 and the collagen receptor CD167a (or Discoidin domain receptor 1; Fig. 2a, i.e. heatmap), validated at the protein level by immunohistochemical analysis, utilizing tissue sections from human MIBC patients (Fig. 2b). Immunohistochemistry revealed a series of human MIBCs (>T2) expressing COL1 in the surrounding stromal regions, with neighboring cancer cells staining positive for cell surface CD167a (Fig. 2b). Additionally, we found that exogenous COL1 treatment of T24 bladder cancer cells significantly enhanced the mRNA expression of CD167a, but not other candidate collagen receptors, nor to the extent of CD167a induction (Supplementary Fig. 1). These findings led us to further investigate CD167a as a collagen receptor during bladder tumorigenesis.

COL1-CD167a signaling enhances MIBC migration and invasion. To further evaluate the biological roles of CD167a, we generated a lentiviral construct driving CD167a gene expression and stably transduced this vector into T24 cells (a weakly metastatic bladder cancer cell line). The endogenous protein level of CD167a 
a
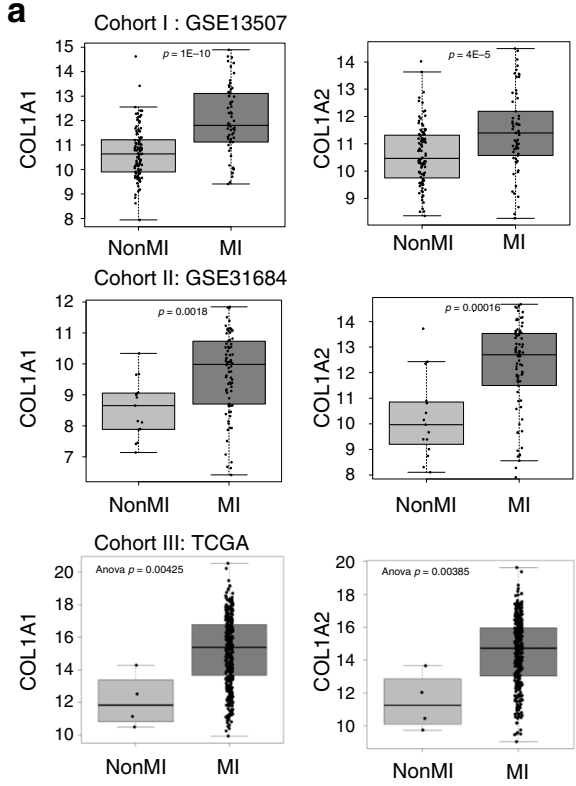

C

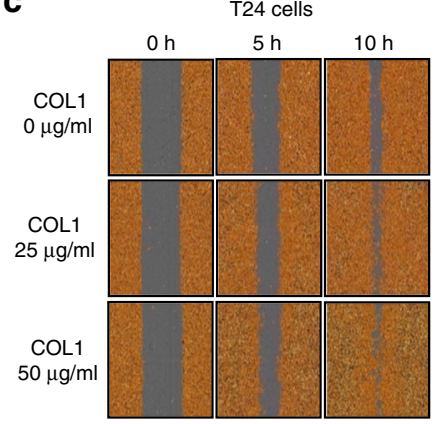

b
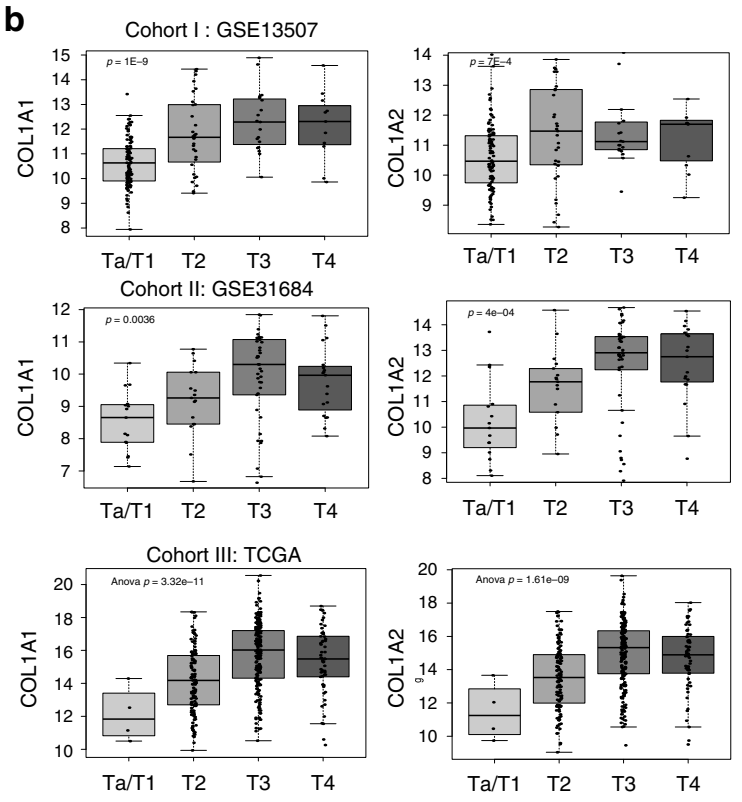

e

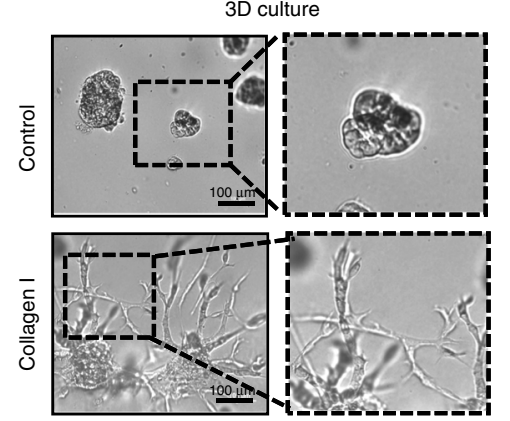

f

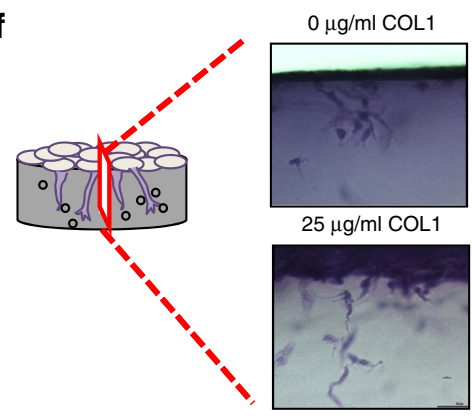

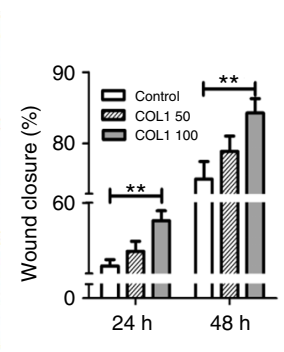

d

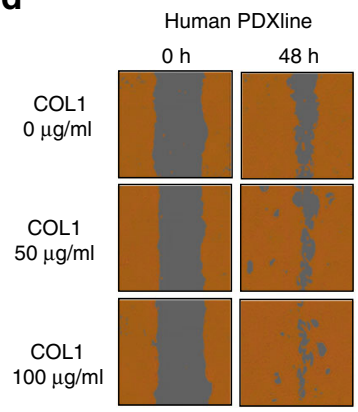

g
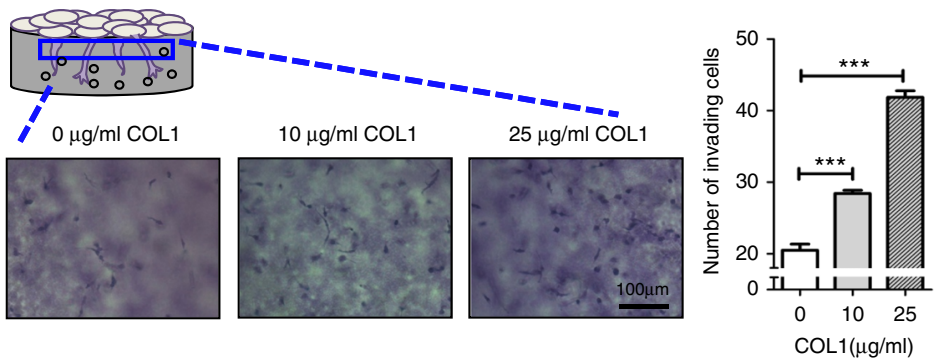

was relatively low in T24 cells, overexpression of CD167a protein was validated by western blot analysis (Fig. 2c). To assess the migratory and invasive ability of CD167a-overexpressing cancer cells, we subjected them to the $2 \mathrm{D}$ cell-scratch assay and the $3 \mathrm{D}$ invasion assay. CD167a overexpression significantly promoted the migration of cancer cells to enhance wound closure (Fig. $2 c, p=$
0.028), and increased cancer cell invasion into 3D matrices (Supplementary Fig. $2 a-c, p<0.001)$. These data confirmed the roles of CD167a overexpression in promoting cell migration and local invasion. Further functional studies revealed a cooperative effect of exogenous COL1 and CD167a receptor expression in an augmented migratory capability of CD167a-overexpressing T24 bladder cancer 
Fig. 1 Collagen I expression correlates with increasing tumor stage and functionally enhances local invasion. a Comparative analysis of collagen I gene expression (COL1A1 and COL1A2) with non-muscle invasive and muscle-invasive bladder cancer status in three different human bladder cancer patient cohorts (cohort I: kim, $n=165^{20}$, cohort II: Riester, $n=93^{21}$, cohort III: TCGA $n=376^{22}$ ). b Correlation of COL1A1 and COL1A2 genes expression with clinical tumor staging (Bladder cancer T staging: pathological evaluation of invasion) in cohorts from $\mathbf{a}$. c Dose-dependent treatment of exogenous collagen $\mathrm{I}\left(0,25\right.$, and $\left.50 \mu \mathrm{g} \mathrm{ml}^{-1}\right)$ and its effects on T24 cell migration. Left panel: Representative images of wound closure at 0,5 , and $10 \mathrm{~h}$ under collagen I treatment. Right panel: Quantification of collagen l-induced percent migration at $10 \mathrm{~h}$ post-wound induction relative to $0 \mathrm{~h}$. $\mathbf{d}$ Dose-dependent treatment of collagen I ( 50 and $100 \mu \mathrm{g} \mathrm{ml}^{-1}$ ) and its effects on a patient-derived xenograft (PDX) culture cell migration. Left panel: Representative images of wound closure at 0 and $48 \mathrm{~h}$ of collagen I treatment. Right panel: Quantification of collagen I-induced percent migration at 24 and $48 \mathrm{~h}$ post-wound induction relative to $0 \mathrm{~h}$. e Representative images of T24 cancer cells cultured in a three-dimensional (3D) matrigel matrix in the absence (top panel, control) or presence (bottom panel) of collagen I $\left(0.25 \mathrm{mg} \mathrm{ml}^{-1}\right)$. $\mathbf{f}, \mathbf{g}$ The 3D-invasive capacity of T24 cells in the presence or absence of collagen I treatment ( 0 , 10 or $25 \mu \mathrm{g} \mathrm{ml}^{-1}$ ) for $48 \mathrm{~h}$. Representative photos of perpendicular (f, left panel) and horizontal sections ( $\mathbf{g}$, left panel) of tumor cells invading through the matrix. The distance and the corresponding number of invading cells from the monolayer into the matrix were quantified as presented in right panel of graft $\mathbf{f}, \mathbf{g}$, respectively. Statistical analysis: $\mathbf{a}, \mathbf{b}$ Analysis of Variance test (ANOVA); $\mathbf{c}-\mathbf{g}$, a two-tailed, unpaired student's $t$-test. Error bar: mean \pm SEM, $n=3$ independent experiments. ${ }^{\star \star} p<0.01,{ }^{\star \star \star} p<0.005$

a

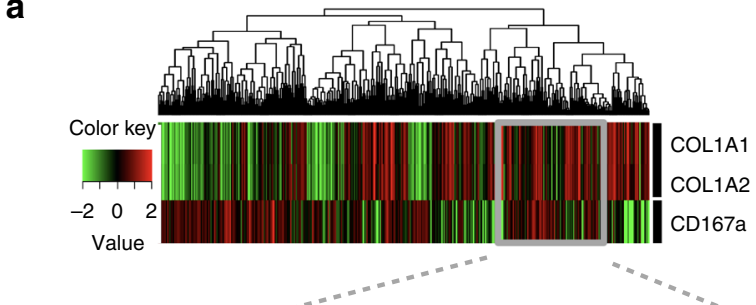

b

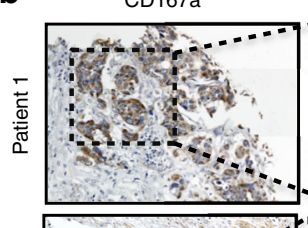

CD167a

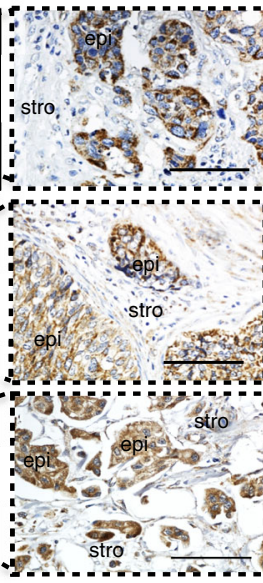

Collagen I

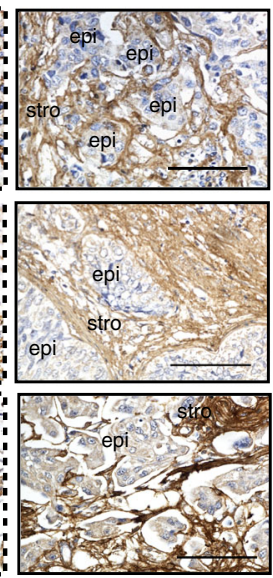

c

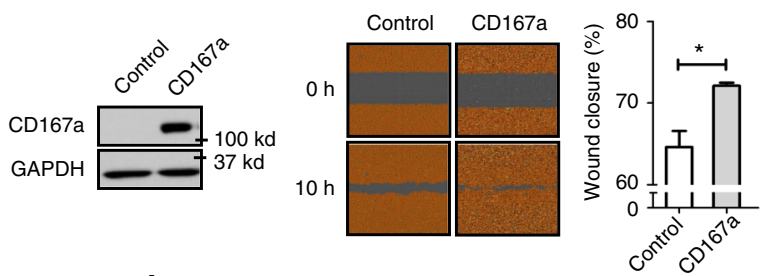

d
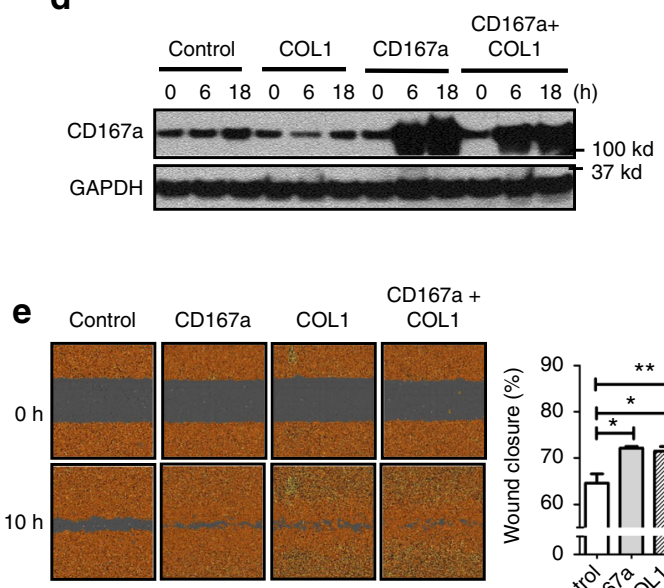

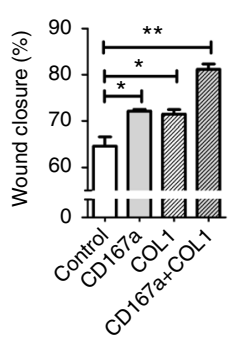

Fig. 2 CD167a expression enhances cell migration in vitro. a Hierarchical clustering of COL1A1, COL1A2 (collagen genes), and CD167a (collagen receptor) genes expression in a human bladder cancer patient cohort (cohort III: TCGA); red and green colors indicate high and low expression, respectively. Grey box indicates patients with co-expression of COL1A1/COL1A2 and CD167a genes. b Immunohistochemical analyses of collagen I and CD167a in representative human MIBC tissues verified the localization of CD167a positive cancer cells in adjacent to stromal collagen I expression. Scale bar:100 $\mu$ m. c Left panel: Western blot analyzing CD167a protein expression in mCherry-CBLuc-Control and mCherry-CBLuc-CD167a-T24 cancer cells. Middle panel: Representative images of mCherry-CBLuc-Control and mCherry-CBLuc-CD167a-T24 cancer cell migration capacity in vitro. Right panel: Quantification of percent migration at $10 \mathrm{~h}$ post-wound induction relative to $\mathrm{O}$ h. d, e Combinatorial effects of exogenous collagen I and CD167a overexpression in cancer cell migration in vitro. Doxycycline-inducible CD167a-expressing T24 cancer cells were subjected to the wound-healing assay with or without collagen I treatment. Cell lysates were harvested after collagen I and doxycycline $(15 \mathrm{ng} / \mathrm{ml})$ stimulation for subsequent western blot evaluation at the indicated time points $(0,6$, and $18 \mathrm{~h})$. Left panel: Representative images of wound closure at 0 and $10 \mathrm{~h}$ upon treatment with $25 \mu \mathrm{g} \mathrm{ml}^{-1}$ collagen I. Right panel: Quantification of percent migration at $10 \mathrm{~h}$ post-wound induction relative to $0 \mathrm{~h}$. Statistical analysis: a two-tailed, unpaired student's $\mathrm{t}$-test. Error bar: mean $\pm \mathrm{SEM}, n=3$ independent experiments. ${ }^{\star} p<0.05,{ }^{\star \star} p<0.01$

cells to initiate wound closure (Fig. 2d, e). Collectively, these findings established a biological role for COL1 to activate CD167a on cancer cells, enhancing cell migration and local invasion within the tumor microenvironment.

CD167a promotes distal lung metastasis in vivo. To further evaluate the in vivo functional roles of CD167a within the metastatic cascade, we engineered the weakly metastatic T24 cancer cells to carry mCherry fluorescence and click-beetle luciferase (CBLuc) reporters for in vivo tracking. We then compared T24 cancer cells overexpressing CD167a to parental cells in their ability to initiate distal lung metastases (Fig. 3a). First, we utilized a spontaneous metastasis model by ectopically inoculating $1 \times 10^{6}$ CD167a-overexpressing or empty-vector 


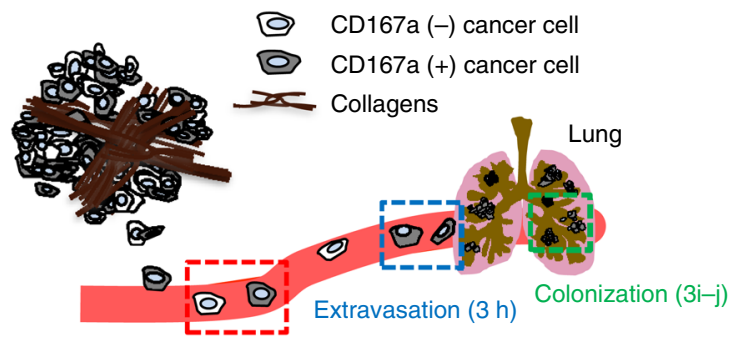

Circulating tumor cells $(3 e-g)$

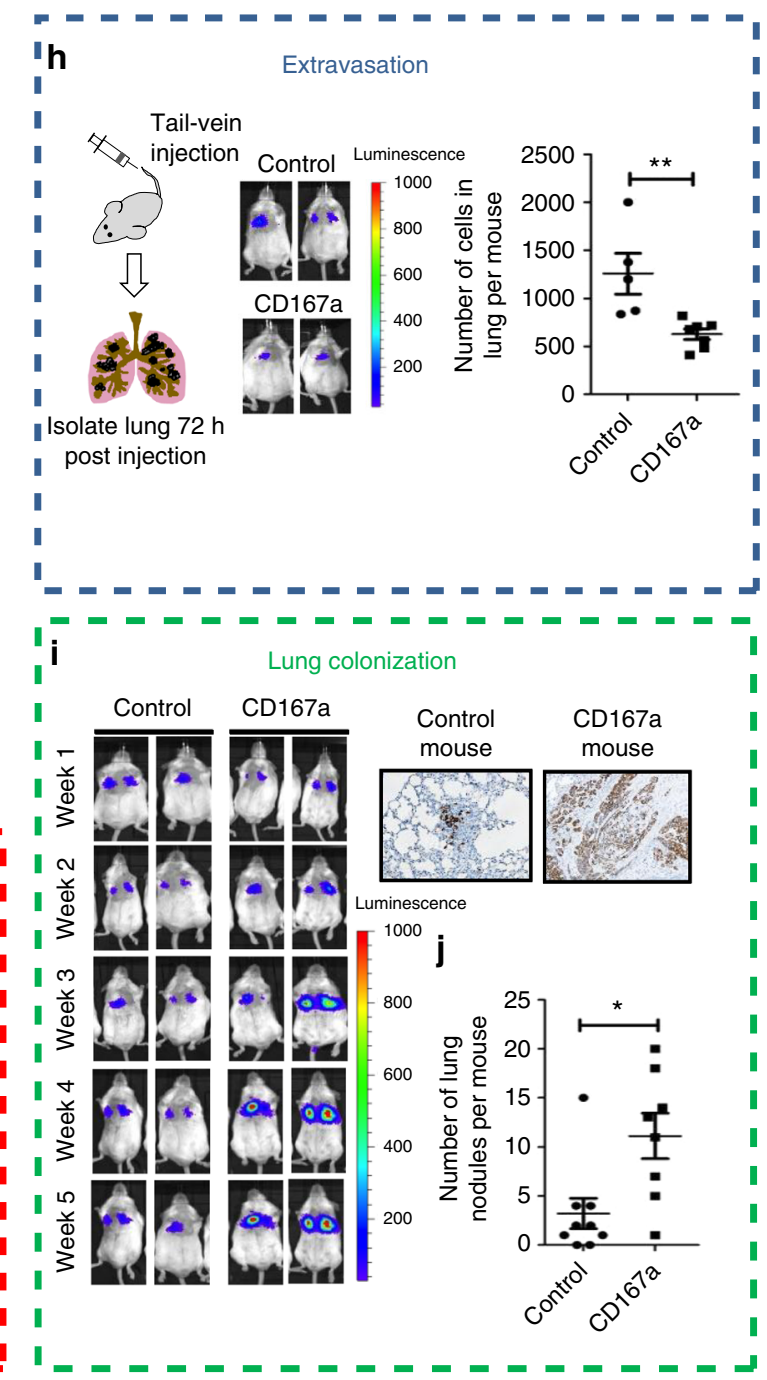

Fig. 3 CD167a facilitates lung colonization in vivo. a Schematic representation of the metastatic cascade: this includes intravasation of tumor cells into the circulation as circulating tumor cells (CTCs) (red box), extravasation of CTCs from the circulation (blue box), and colonization at distant organ, e.g. lung (green box). b Endpoint analysis of mouse weight ectopically injected with control or CD167a-overexpressing T24 cancer cells $(n=7)$ over a 12-week period. c Quantification of metastatic nodules in lung of mice injected with control or CD167a-overexpressing T24 cancer cells $(n=7)$. d Quantification of metastatic nodule total area $\left(\mathrm{mm}^{2}\right)$ in lungs of mice injected with control or CD167a-overexpressing T24 cancer cells $(n=7)$. e-g The examination of CTCs frequency in a spontaneous metastatic model. Peripheral blood was collected from mCherry-CBLuc-control or CD167a-overexpressing mCherryCBLuc-T24 cells tumor-bearing mice and analyzed by flow cytometry to quantify fluorescent CTCs. Frequency of single and cluster CTCs is shown in $\mathbf{f}, \mathbf{g}$, respectively. $n=9$. $\mathbf{h}$ Left panel: Schematic illustration of the tail-vein injection model. Middle panel: Representative bioluminescence images (BLI) of mice injected with control or CD167a-overexpressing mCherry-CBLuc-T24 cancer cells. Right panel: Quantification of cancer cell extravasation in the lungs of mice injected with control $(n=5)$ or CD167a-overexpressing mCherry-CBLuc-T24 cancer cells $(n=7) 72 \mathrm{~h}$ post-injection after immunohistochemical analysis. i Left panel: Representative bioluminescence images of mice injected with mCherry-CBLuc-control or mCherry-CBLuc-CD167a-overexpressingT24 cancer cells via tail-vein, temporally tracked for tumor colonization up to 5 weeks post injection (Control $n=9, C D 167 a, n=8$ ). $\mathbf{j}$ Histological lung sections from mice injected with control and CD167a cancer cells from $\mathbf{i}$ were independently analyzed for graphical quantification of metastatic nodules in the lung. Statistical analysis: a two-tailed, unpaired Student's t-test. Error bar: mean \pm SEM. ${ }^{*} p<0.05,{ }^{* *} p<0.01$

transduced T24 cancer cells into Rag2 $2^{-1-}$; IL-2R $\gamma \mathrm{c}^{-/-}$immunocompromised mice, and monitored secondary organ metastasis over time using IVIS imaging. At $\sim 4-6$ weeks post tumor inoculation, mice harboring CD167a-overexpressing xenograft tumors started exhibiting a notable loss in body weight when compared to vector controls, which became statistically significant at 10-12 weeks (Fig. 3b, control $n=7$, CD167a $n=7$ ). Since one of the clinical symptoms of metastatic cancer patients is unintended weight loss, and taking into consideration that T24 cancer cells could form lung metastasis ${ }^{24}$, we speculated the unintended weight loss observed in the CD167aoverexpressing group likely resulted from accelerated distal metastases. We therefore analyzed the gross morphology and histopathology of lung tissues collected from both experimental groups (Fig. 3c, d, control $n=7$, CD167a $n=7$ ). At the time of sacrifice, histologic analysis by H\&E staining uncovered microscopic metastases in both groups; interestingly, there was a statistically significant increase in the number (Fig. 3c) and size (Fig. 3d) of metastatic lung nodules formed from CD167a-overexpressing T24 cells, when compared with parental cells in this spontaneous metastatic model. Collectively, these findings revealed a role for CD167a in enhancing the development of distal lung metastases in an human bladder cancer model in vivo. 
CD167a does not impact tumor cell intravasation. To mechanistically dissect the roles of CD167a during the metastatic cascade (Fig. 3a), we first evaluated its capacity to facilitate tumor intravasation into the circulation as CTCs (Fig. 3e). The analyses of CTCs were performed using the spontaneous metastatic model, comparing mice ectopically injected with CD167a-overexpressing $(n=9)$ or vector transduced T24 cells $(n=9)$. After $\sim 8-9$ weeks post tumor inoculation when tumor volume reached approximately $100 \mathrm{~mm}^{3}$, peripheral blood was collected and subjected to flow cytometric analysis (Fig. 3e). Since we engineered these cancer cells to carry the fluorescent protein mCherry, we could readily detect CTCs in peripheral blood via their fluorescent intensity using flow cytometry (Supplementary Fig. 3). Interestingly, there were no statistically significant difference in the number of CTCs detected as single cells between the two groups (Fig. $3 \mathrm{f}$; Control group $=0-371$ CTCs, CD167a group $=3-448$ CTCs). A recent report illustrated CTC clusters possess greater metastatic potential than single CTCs ${ }^{27}$; therefore, we further evaluated the occurrence of CTC clusters. Nonetheless, the frequency of CTC clusters between the two groups was not significantly altered (Fig. $3 \mathrm{~g}$; Control group $=0-381$ CTCs, CD167a group $=1-99$ CTCs). Together, these findings revealed tumor intravasation into the circulation was unlikely a primary mechanism for CD167a to enhance distal metastasis.

CD167a does not enhance extravasation to mediate metastasis. Next, to address whether CD167a played a role in regulating extravasation from the circulation into lung, we employed a standard tail-vein injection model (Fig. $3 \mathrm{~h})^{28}$. The tail-vein injection assay is a relevant experimental metastasis model to investigate extravasation, since blood vessels in lung are the first capillary bed encountered by cancer cells after injection. Briefly, $1 \times 10^{6}$ CD167a-overexpressing or vector transduced T24 cells were injected into the tail-vein, allowing temporal tracking of extravasation into lung tissues (Supplementary Fig. 4a). The efficiency of tumor cell extravasation into lung tissues was quantified at $72 \mathrm{~h}$ post injection (control $n=5$, CD167a $n=7$ ), via bioluminescence imaging (BLI) (Fig. 3h), and IHC staining (Supplementary Fig. 4b). Intriguingly, while CD167a-expressing cancer cells were more efficient in forming distal lung metastases, they were not as efficient in extravasating into lung tissues at this early time point (Fig. $3 \mathrm{~h}$ ), revealing extravasation was not a major mechanism responsible for their enhanced metastasis.

CD167a enhances metastatic lung colonization. To further evaluate metastatic lung colonization in vivo, the tail-vein metastasis assay was repeated (Fig. 3j, and Supplementary Fig. 4a), and bioluminescent activity was longitudinally tracked by IVIS imaging weekly to monitor lung colonization over a 5 week period. While both groups exhibited a temporal increase in bioluminescent signal (Fig. 3i, control $n=9$, CD167a $n=8$ ), a notable increase of signal intensity in the CD167a-overexpressing group was observed as early as 3 weeks post injection, which became statistically significant at week 5 during the time of sacrifice (Fig. 3i). Detailed histologic analysis confirmed a significant increase in the number of metastatic foci in mice inoculated with CD167a-overexpressing bladder cancer cells, when compared with empty vector transduced group (Fig. 3j); thus, validating a role for CD167a in enhancing metastatic colonization in lung.

Airway smooth muscle cells as a metastatic niche in lung. Another intriguing finding was uncovered by detailed pathological analysis of corresponding metastatic foci via H\&E staining. While empty vector transduced cells formed typical metastatic nodules within alveolar spaces (Fig. 4a, * indicates alveolar space), the morphology and colonization pattern of CD167aoverexpressing metastatic foci were noticeably different (Fig. 4b, arrows outlining the areas of metastatic cancer). A notable fraction of CD167a-expressing metastatic foci uniquely resided along or within airway smooth muscle cells (ASMCs), representing an original and newly-described colonization site for metastatic foci (Fig. 4b). Additional examples of these unique metastatic foci resembling early colonization (Supplementary Fig. 5a, b) or overt lung metastases (Fig. $4 \mathrm{~b}$ and Supplementary Fig. 5c-i) were presented, with their proximal localization adjacent to, or within ASMCs, respectively. Quantification of these two types of metastatic foci indicated a statistical significance $(p<0.0001)$, with CD167a-expressing foci preferentially localized to ASMCs (Fig. 4c, control $n=7$, CD167a $n=7$ ). To validate their unique localization within ASMCs, an antibody against alpha smooth muscle actin ( $\alpha$-SMA) was used to outline ASMCs by immunofluorescence staining (Fig. 4d; red color, and Supplementary Fig. 5e, f), and anti-CD167a was used to mark the relative localization of metastatic cancer cells (Fig. 4d; green color, and Supplementary Fig. 5e, f). Immunofluorescence costaining validated the colonization of CD167a-expressing cancer cells (Fig. 4d, green) within a-SMA(+) airway smooth muscle cells (Fig. 4d, red), confirming H\&E staining results in serial section (Fig. 4e, f, higher magnification). To our knowledge, ASMCs had not been reported to function as supporting niche cells in cancer-related studies. Independently, ASMCs were reported to mediate ECM remodeling by depositing collagen in asthma research studies ${ }^{29,30}$, where type III collagen (COL3) was reported to be the predominant collagen expressed in lung ${ }^{31,32}$. Since COL3 could also act as a ligand for CD167a $\mathrm{a}^{33,34}$, it led us to hypothesize that ASMCs might be a source of collagen, thus providing a supportive niche for the colonization of CD167aexpressing cancer cells. To evaluate this, immunohistochemical staining of CD167a and COL3 were performed in serial sections (Fig. 4g, h), revealing metastatic cancer cells residing within ASMCs (Fig. 4g) are abundantly surrounded by COL3 (Fig. 4h, i). Such observations extended to metastatic foci representing early colonization, i.e. small clusters of cancer cells aligning adjacent to COL3-rich ECM (Supplementary Fig. 5 area I:a, b, and area II:c-f), and larger nodules of metastatic cancer masses entirely surrounded by COL3 positive areas (Supplementary Fig. $5 g-\mathrm{i}$, area III).

To establish the generalization of airway smooth muscle cells as a metastatic niche, we evaluated additional models of lung metastases (Fig. 4j-s). These included a selected model of highly metastatic T24 subline (Fig. $4 \mathrm{j}-\mathrm{m}, n=5$ ), and a human patient-derived xenograft that spontaneously metastasized to lung (Fig. $4 \mathrm{n}-\mathrm{q}, n=4$ ). The highly metastatic subline (T24-LungMet) was generated from parental T24 cancer cells by serially re-injecting five consecutive times through tail-vein in vivo (Fig. 4j). IVIS imaging (Fig. 4j) and $\mathrm{H} \& \mathrm{E}$ staining (Supplementary Fig. 6) indicated its enhanced metastatic capacity to colonize lung tissues. Using CD167a staining to outline metastatic cancer cells, and a-SMA to outline ASMCs, we identified prevalent metastatic foci localized within COL3-rich ASMCs from the T24-LungMet model (Fig. 4j-m), as well as the spontaneous lung metastatic model of human PDX (Fig. $4 \mathrm{n}-\mathrm{q}$ ). Further, western blot analysis of CD167a protein level in these three independent lung metastatic models (Fig. 4r) revealed an association to the frequency of metastatic foci to airway smooth muscle cells (Fig. 4s), Taken together, these results illustrated airway smooth muscle cells as a newly-identified metastatic niche within the lung microenvironment.

ASM cells secret collagen III to enhance colonization. To investigate whether ASMC-secreted host factors could influence colonization in lung, we collected conditioned media from human 
CD167a (+) metastatic cancer cells
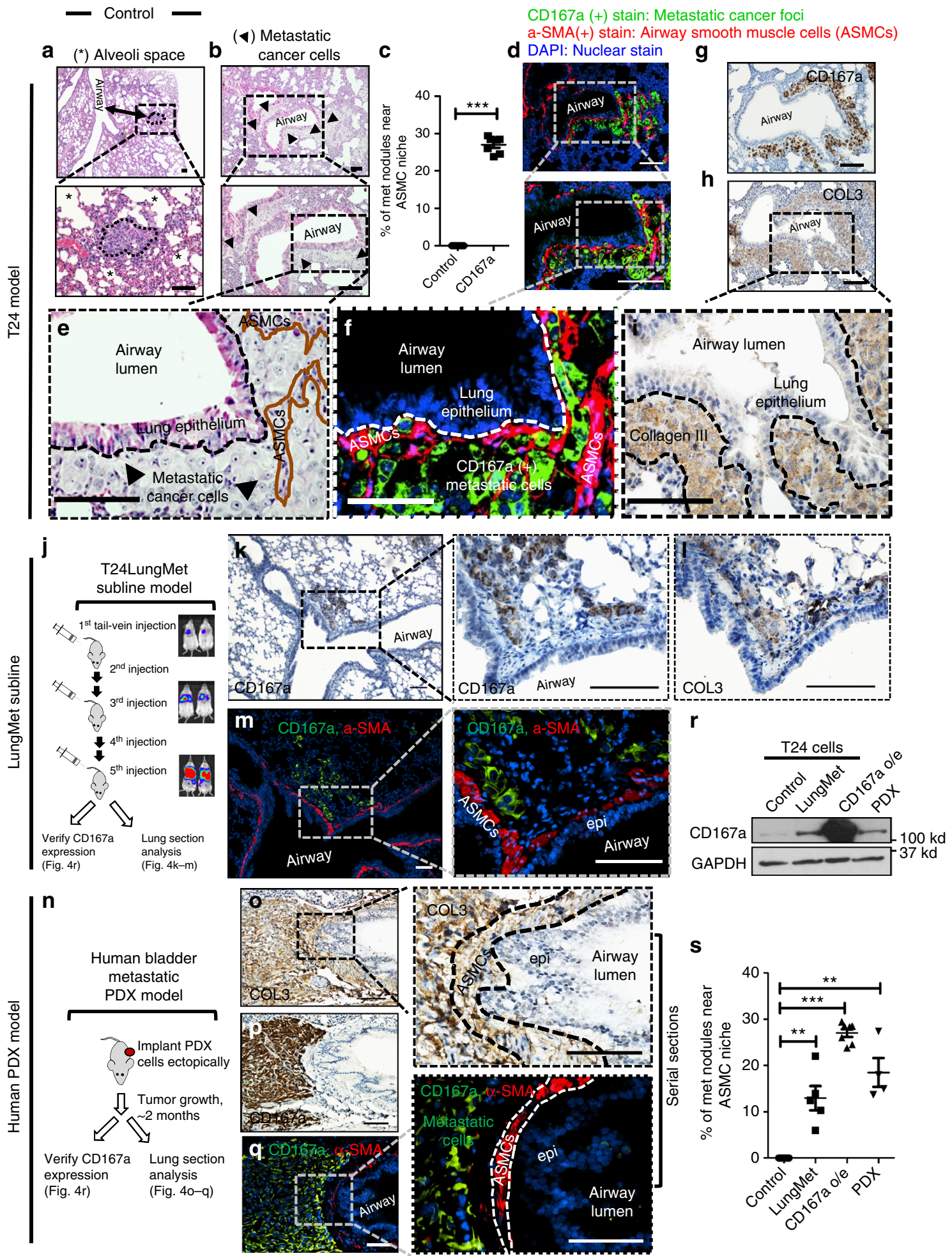

$\mathbf{s}$

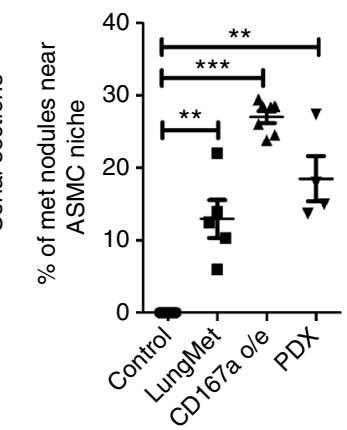

ASMCs and evaluated its effects in altering the colony-formation capacity of tumor cells, utilizing the soft agar assay (as a surrogate for lung colonization; Fig. 5a). Both the T24 cells and human bladder PDX culture were selected as representative models (Fig. 5b, c). Conditioned media from ASMCs significantly enhanced the frequency of colony formation in parental T24 cells (Fig. 5b, open boxes), and even more so in the CD167a-overexpressing T24 cells (Fig. 5b, grey boxes). A similar finding was observed in the human metastatic PDX model, as ASMC-conditioned media significantly enhanced the frequency and size of colony formation (Fig. 5c). Since our primary emphasis was collagen secretion from ASMCs as the putative ligand for CD167a, and prior studies revealed COL3 as a predominant collagen within lung tissues, we analyzed the relative mRNA and protein expression of COL3 in cultured human ASM cells. COL3 mRNA expression was markedly higher in 
Fig. 4 Airway smooth muscle cell (ASMC) is a metastatic niche in the lung. a H\&E staining of lung sections revealing typical metastatic lung foci within alveolar spaces that were formed from vector control T24 cancer cells. $\left(^{\star}\right)$ denotes alveolar spaces. Scale bar:100 $\mu \mathrm{m}$. b H\&E staining of lung sections revealing morphologically distinct metastatic foci residing adjacent to ASMCs (outlined by arrows), which formed from CD167a-overexpressing T24 cancer cells. Scale bar: $100 \mu \mathrm{m}$. c Quantification of unique lung metastases with proximal ASMCs localization (control, $n=7 ;$ CD167a, $n=7$ ). d Immunofluorescent co-staining of $\alpha$-SMA (an ASMCs marker, red), and CD167a (to label metastatic cancer cells, green), in a serial section to b. Scale bar:100 $\mu \mathrm{m}$. e-i Serial sections demonstrating H\&E staining, IF and IHC staining of various markers illustrating the colonization of CD167a-expressing cancer cells within COL3rich ASMCs. Anti-CD167a antibody was used to outline metastatic cancer cells, anti- $\alpha$-SMA and anti-collagen III antibodies were used to label ASMCs and secreted collagens, respectively. Scale bar:100 $\mu \mathrm{m}$. j Serial transplantation scheme to establish a highly-metastatic model (T24-LungMET) that efficiently colonized into lung tissues, by serially transplanting parental T24 cells into tail vein five consecutive times, monitored by bioluminescence imaging. k, I Representative serial sections of metastatic foci stained with anti-CD167a to mark metastatic cancer cells and its relative localization to COL3 in T24-LungMET model. $\mathbf{m}$ Immunofluorescence co-staining images of CD167a (green) and $\alpha$-SMA (red) showing the relative localization of CD167a + metastatic cancer cells and their proximity to $\alpha$-SMA+ ASMCs. Scale bar:100 $\mu \mathrm{m}$. $\mathbf{n}$ Experimental scheme to establish a spontaneous human bladder metastatic PDX model. o, p Representative serial sections of metastatic foci stained with anti-CD167a to mark metastatic cancer cells and their proximity to COL3. q Immunofluorescence co-staining images of CD167a (green) and $\alpha$-SMA (red) showing the relative localization of CD167a+metastatic cancer cells and their proximity to $\alpha$-SMA+ASMCs. Scale bar:100 $\mu \mathrm{m}$. $\mathbf{r}$ Western blot analysis comparing CD167a protein expression in parental T24 cells, T24-LungMET model, CD167a-overexpressing T24 model and the human bladder metastatic PDX model. s Quantification of lung metastases proximal to ASMCs, in the metastatic bladder carcinoma models evaluated in $\mathbf{r}$. Statistical analysis: a two-tailed, unpaired student's t-test. Error bar: mean \pm SEM. ${ }^{\star *} p<0.01,{ }^{* \star *} p<0.005$

ASM cells than in a commercially available human cDNA mix or T24 cancer cells (Fig. 5d). Similarly, COL3 protein was abundantly expressed within ASM cells as demonstrated by western blot analysis (Fig. 5e). Additionally, COL3 was detected as secreted protein peptides in the ASMC conditioned media via targeted mass spectrometry (Fig. 5f). Collectively, these results verified COL3 as a secreted protein by ASM cells (Fig. $4 \mathrm{f}$ and Supplementary Fig. 7), substantiating the tissue level results from Fig. 4.

To evaluate whether COL3 could recapitulate the effects of ASMC-conditioned media in impacting colony formation, COL3 was embedded in the top layer of the soft agar matrices with tumor cells. Intriguing, COL3 phenocopied the effects of ASMCconditioned media, which increased colony formation significantly in control and CD167a-overexpressing T24 cancer cells (Fig. 5g), as well as the metastatic PDX (Fig. 5h). Collectively, these results confirmed a role for ASMCs and COL3 in promoting colony formation.

COL3-CD167a-Stat3 axis in the lung microenvironment. To identify the downstream mediator for the COL3-CD167a axis, we evaluated our newly established metastatic T24-LungMet subline from Fig. 4j. Enhancement of metastatic potential in this T24LungMet subline associated with a significant increase in CD167a protein expression, which corresponded with an elevated phosphotyrosine level of Stat3 at a.a. pY705 (Fig. 6a); thus, implicating a molecular link. To evaluate whether ASMCs/COL3 goes through a CD167a-Stat3 axis, we first examined whether ASMC-derived conditioned media or exogenous COL3 treatment had any effects in modulating Stat3 phospho-tyrosine (pY705) level. Both treatment conditions enhanced phospho-tyrosine Stat3 level in the T24 bladder cancer and the human PDX model (Fig. 6b, c), which was significantly diminished by a CD167a/DDR1 selective kinase inhibitor (DDR1-IN-1) (Fig. 6b, c). Since the DDR1-IN-1 compound was originally discovered as a selective kinase inhibitor that blocks CD167a/DDR1 autophosphorylation ${ }^{35}$, these results connected Stat3 as a downstream molecule to the COL3-CD167a canonical axis. Alternatively, a recent report revealed CD167a (or DDR1) can mediate signals via a non-canonical pathway in breast cancer metastases 36 . Our results using the selective kinase inhibitor supported a canonical kinase-dependent role of CD167a/DDR1 in activating Stat3 in our bladder cancer metastasis models (Fig. 6b, c), highlighting the significance of organ-site specificity, postulating discrete canonical and non-canonical CD167a/DDR1 signaling in different tissue types.
To uncover a mechanistic link, we performed a proteomics screen by coupling co-immunoprecipitation with mass spectrometry (IP-MS) ${ }^{37}$ to pull down and categorize CD167a-interacting proteins. Interestingly, HSP90 and CDC37, two proteins forming a chaperone complex, are identified among the top of the list (Fig. 6d). HSP90 functions as a molecular chaperone that modulates its client proteins to impact their protein folding, stability and activation ${ }^{38}$. Hence, HSP90 complexes participate in various biological functions, and signal transduction. Furthermore, it has been reported that Stat 3 is also a client protein of HSP90 ${ }^{39,40}$, therefore, implicating CD167a/HSP90 as a candidate regulator of Stat 3 activation in our cancer model.

To further explore this mechanistic link of CD167a-HSP90Stat3, we performed reciprocal co-IP using an anti-HSP90 antibody to pull down its interacting protein complexes in control and CD167a-overexpressing T24 cells. Our co-IP results validated the IP-MS profiling results in Fig. $6 \mathrm{~d}$, clearly showing protein complex formation between CD167a and HSP90 in our cancer model (Fig. 6e, left panel). Further, Stat3 tyrosine phosphorylation indicative of its activity is increased in CD167aoverexpressing T24 cells (Fig. 6e, right panel), closely associated with an increased complex formation between CD167a and HSP90 (Fig. 6e, left panel). To further evaluate whether this CD167a/HSP90 complex formation is dependent on CD167a kinase, the selective CD167a/DDR1 inhibitor (DDR1-IN-1) was used and found to partially disrupt this complex formation (Fig. 6f, g). Since HSP90 was reported elsewhere to be a regulator for Stat $3^{39,40}$; accordingly, we explored whether disruption of HSP90 function by its potent inhibitor, Geldanamycin, might affect the tyrosine phosphorylation of Stat3. Geldanamycin effectively disrupted the interaction of HSP90 with CD167a in the T24 model (Fig. 6f), and convincingly diminished ASMCinduced Stat 3 tyrosine phosphorylation in a dose-dependent manner, both in T24 bladder cancer (Fig. 6h) and the human PDX models (Fig. 6i). Similarly, COL3-induced Stat3 tyrosine phosphorylation was also significantly abrogated by the HSP90 inhibitor (Fig. 6j, k). Collectively, these results uncovered CD167a-HSP90 as a newly-identified interacting protein complex, which connected Stat3 as a downstream molecule to the CD167a canonical axis in bladder cancer.

Targeting Stat3 abrogates metastatic colonization in lung. Since DDR1-IN-1 is a compound not proven for pharmacological application in vivo and HSP90 is a molecular chaperone with many known client proteins additional to Stat3, we decided to place 
a

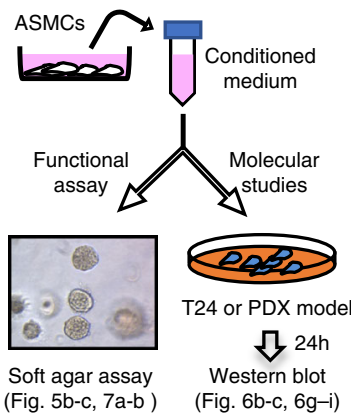

d

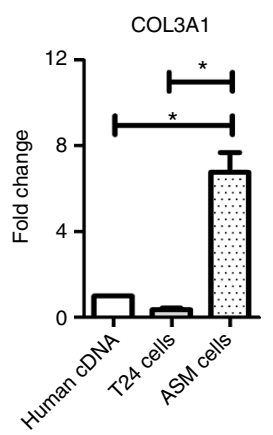

e

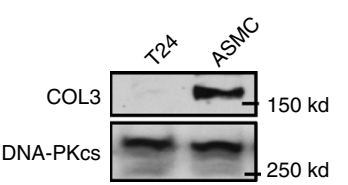

g

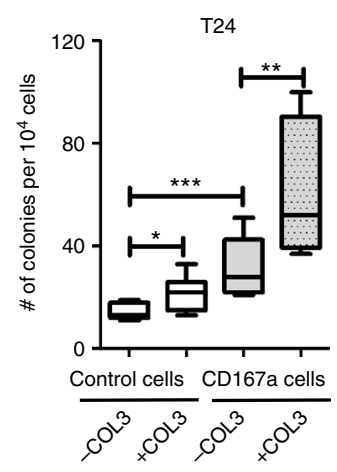

b

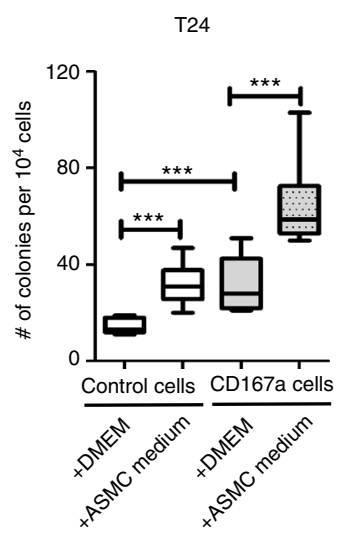

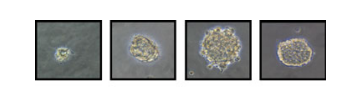

C
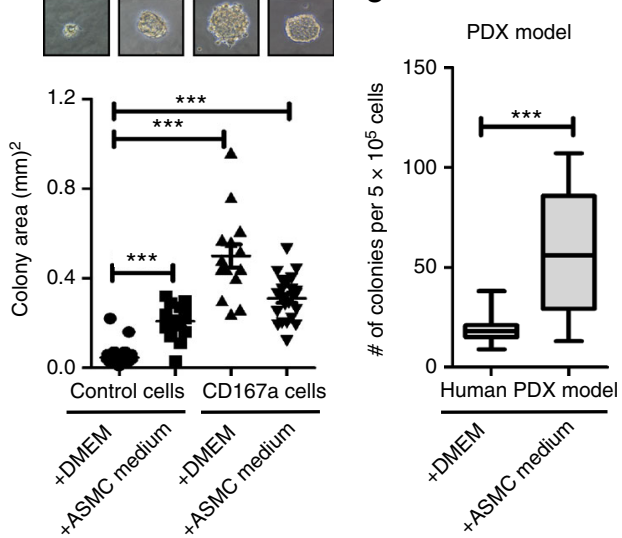

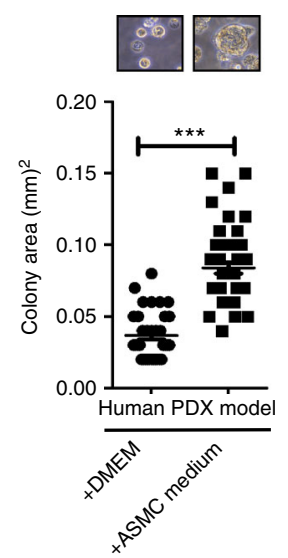

$f$ COL3 peptide peak:

(1)

$0 \mathrm{~h}$

(3) INTDEIMTSLK
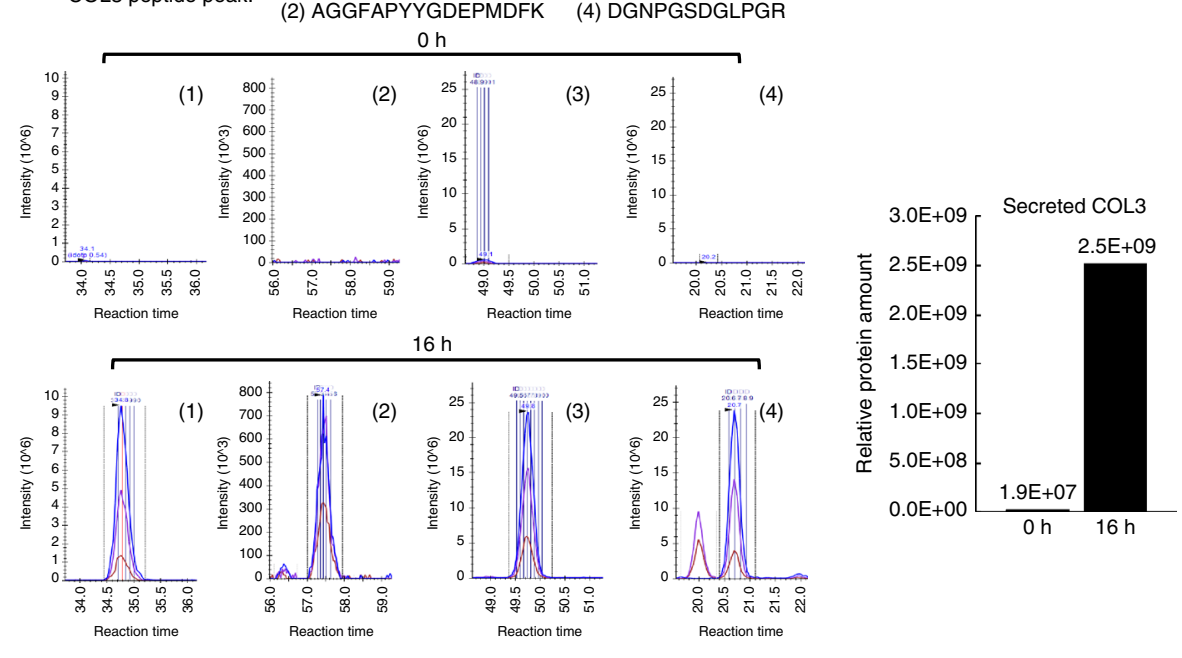

h
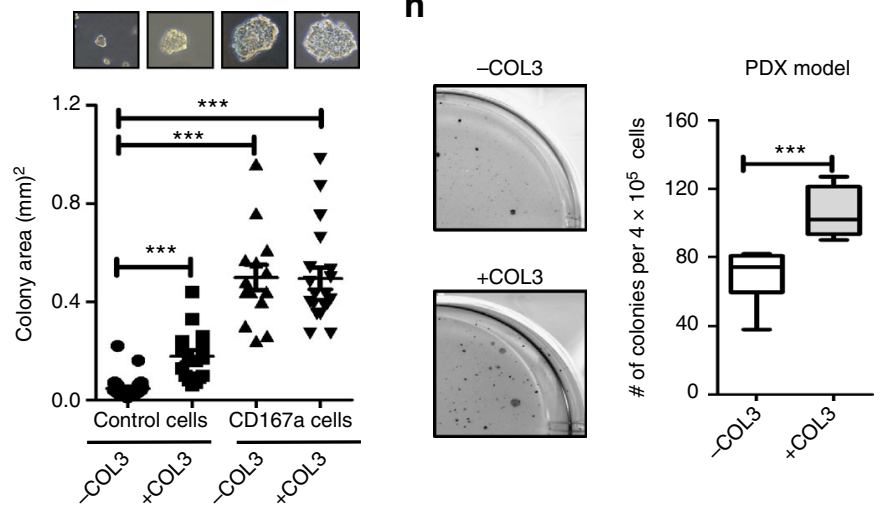

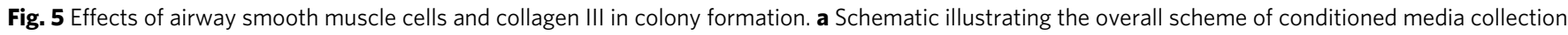
from airway smooth muscle cells (ASMCs) for the evaluation of its impact on colony formation in vitro, and subsequent molecular analysis.

b Quantification and size measurement of colonies formed from control and CD167a-overexpressing T24 cancer cells, and c a human metastatic PDX model in a classical soft-agar assay, in the presence or absence of ASMC conditioned media over a 14-day period. Representative phase-contrast images depict the relative colony size from respective conditions. d Quantitative real-time qPCR analysis of COL3A1 mRNA in cultured human ASMCs. Human cDNA mix and cultured T24 cells were harvested as controls. GAPDH was used as reference and the relative expression was normalized against human cDNA mix. e Western blot analysis of COL3 expression in human ASMCs and T24 cancer cells. DNA-PKcs was used as the loading control. $\mathbf{f}$ Targeted mass spectrometry analysis to evaluate protein peptides specific toward COL3, that were secreted into the supernatant or conditioned media collected from cultured human ASMCs at $\mathrm{O}$ and $16 \mathrm{~h}$ after incubation. The amount of COL3 was calculated based on MS1 signal area under curve using SKYLINE software based on top four strongly detected peptides. $\mathbf{g}$ Colony formation assay of control and CD167a-overexpressing T24 cancer cells upon COL3 treatment $\left(50 \mu \mathrm{g} \mathrm{ml}^{-1}\right)$. Number and size of colonies are shown on the left and right panel, respectively. $\mathbf{h}$ Colony formation assay of the human metastatic

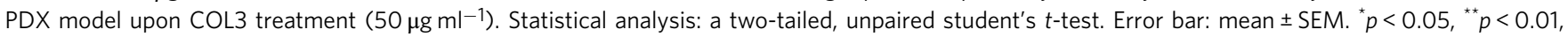
${ }^{\star \star \star} p<0.005$ 
a

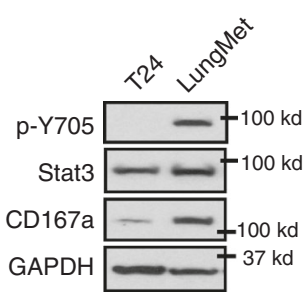

b

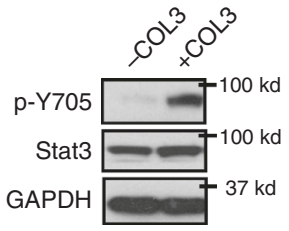

T24 model

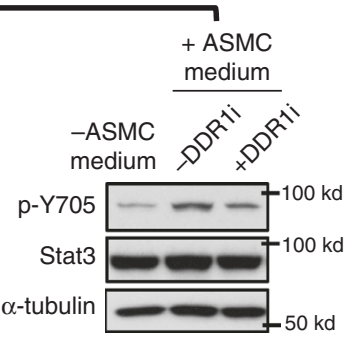

C

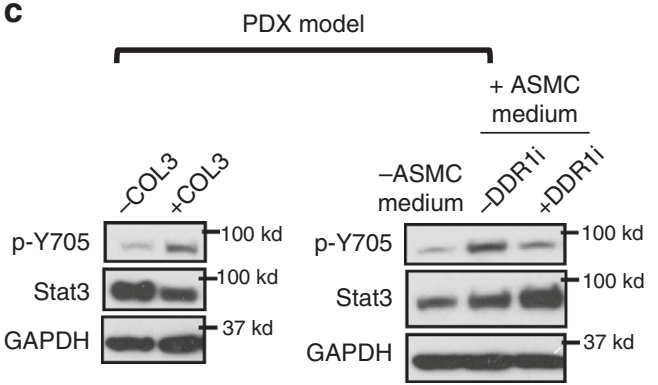

d

\begin{tabular}{|c|c|c|c|c|c|}
\hline \multirow[b]{2}{*}{ GeneSymbol } & \multicolumn{2}{|c|}{ Control } & \multicolumn{2}{|c|}{ CD167a IP } & \multirow{2}{*}{$\begin{array}{l}\text { Fold enrichment } \\
\text { CD167a IP/ Control }\end{array}$} \\
\hline & 1 & 2 & & 2 & \\
\hline CD167a & n.d. & n.d. & 186 & 320 & $\infty$ \\
\hline HSP90AB1 & 5.7 & 84.1 & 572 & 716 & 14.3 \\
\hline HSP90AA1 & 1.9 & 28.9 & 243 & 319 & 18.2 \\
\hline CDC37 & 0.02 & 2.7 & 76.2 & 189 & 97.5 \\
\hline
\end{tabular}

e

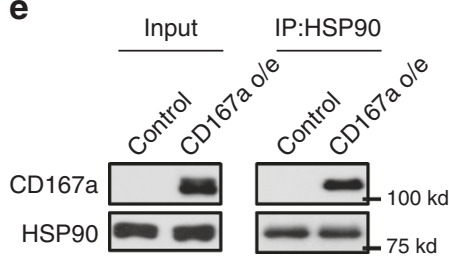

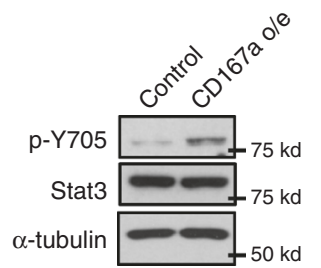

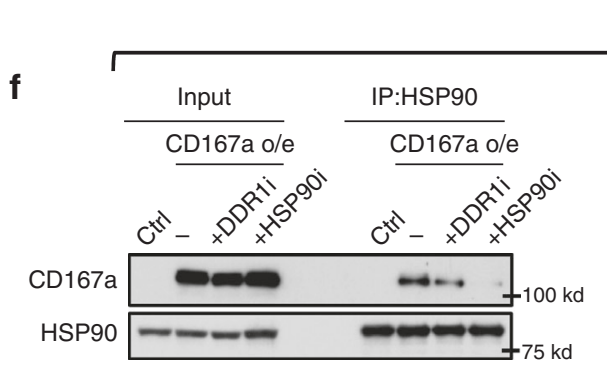

T24 model
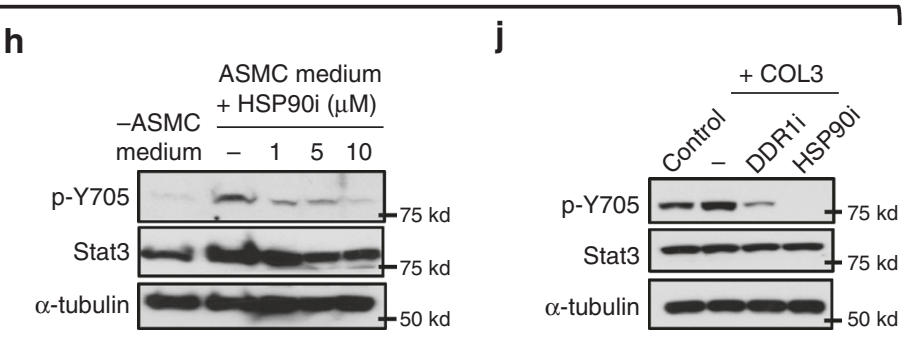

PDX model

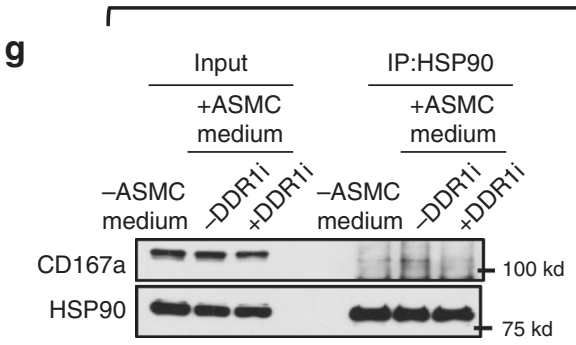

i

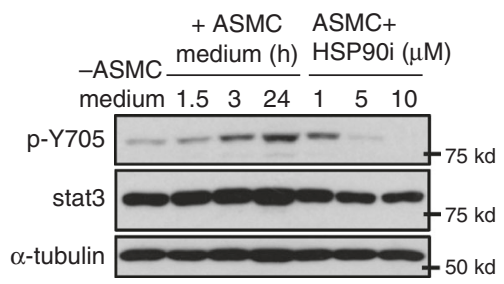

k

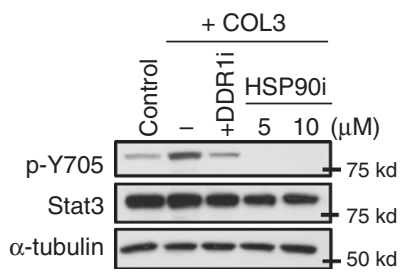

Fig. 6 CD167a-Stat3 axis in the COL3-rich airway smooth muscle cell metastatic niche in lung. a Western blot evaluating the relative protein expression of CD167a, Stat3 and phosphotyrosine Stat3 (Y705) in parental T24 cells and our newly-established metastatic model (T24-LungMET). b, c Effects of COL3, and ASMC-conditioned media and CD167a/DDR1 kinase inhibitor (DDR1-IN-1; 10 $\mu$ M) on Stat3 and phosphotyrosine Stat3 protein expression, in T24 and PDX models. d Coupling CD167a co-IP with mass spectrometry to discover CD167a-interacting partners. Selected CD167a associated gene protein products (GPs) and their corresponding iBAQ values are listed. e Coupling co-IP and western blot to evaluate CD167a-HSP90 interaction, via co-IP using anti-HSP90 antibody followed by western blotting of CD167a in control and CD167a-overexpressing T24 cancer cells. f Effects of CD167a kinase inhibitor (DDR1-IN-1, 10 $\mu \mathrm{M}$ ) and HSP90 inhibitor (Geldanamycin, $5 \mu \mathrm{M}$ ) on CD167a-HSP90 complex formation. Control cells and CD167a-overexpressing T24 cancer cells were treated with COL3 in the absence or presence of indicated inhibitors for $24 \mathrm{~h}$. Cell lysates were collected for co-immunoprecipitation assay using anti-HSP90 antibody. $\mathbf{g}$ PDX cells were cultured in control (-ASMC) or ASMCs conditioned media (+ASMC) with or without DDR1-IN-1 inhibitor $(10 \mu \mathrm{M})$ for $24 \mathrm{~h}$. Cell lysates were assessed for CD167a-HSP90 interaction by co-immunoprecipitation assay followed by western blot analysis. $\mathbf{h}$, i Western blot analysis of Stat3 tyrosine phosphorylation upon treatment with ASMC-conditioned medium in the presence or absence of HSP9O inhibitor (+HSP9Oi, 1, 5, and $10 \mu \mathrm{M}$ ) in T24 and PDX models. j, k Western blot analysis of COL3-induced Stat3 tyrosine phosphorylation in T24 and PDX models upon treatment with CD167a/DDR1 kinase specific inhibitor (DDR1-IN-1, 10 $\mu$ M) and Geldanamycin (HSP90i, $5 \mu \mathrm{M})$

emphasis onto targeting the CD167a-HSP90 downstream effectorStat3, with drugs emergent in late phase clinical trials. We utilized Napabucasin, a small molecule inhibitor originally discovered with selective specificity toward Stat $3^{41}$. Napabucasin (BBI-608) is currently approved by the FDA as an anti-cancer drug in use for Phase II/III clinical trials (https://www.cancer.gov/about-cancer/ treatment/clinical-trials/intervention/napabucasin) and had been shown to prolong the survival of patients with colorectal cancer tumors expressing Stat $3^{42}$. To evaluate whether drug targeting of Stat3 could abrogate ASMC-induced colony formation,
Napabucasin was evaluated and significantly suppressed ASMCinduced colony formation capacity of cancer cells (Fig. 7a, b). Moreover, to evaluate the in vivo efficacy of Napabucasin in modulating lung colonization, we adopted the tail-vein injection model (as in Fig. $3 \mathrm{~h}-\mathrm{i})$. Mice were treated with Napabucasin $(n=6)$ or vehicle as control $(n=6)$ at $72 \mathrm{~h}$ after injection of cancer cells (when cancer cells started lung colonization-Fig. 3h). Napabucasin effectively and significantly abolished metastatic lung colonization, when compared to vehicle treated control mice, in two independent metastatic models of bladder carcinomas (Fig. 7c). Furthermore, 
a

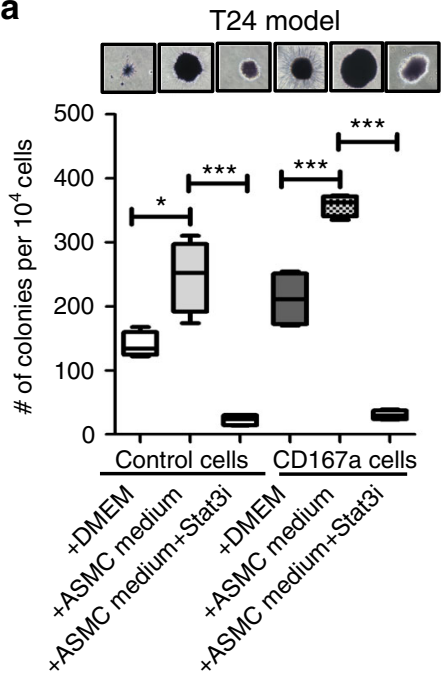

b

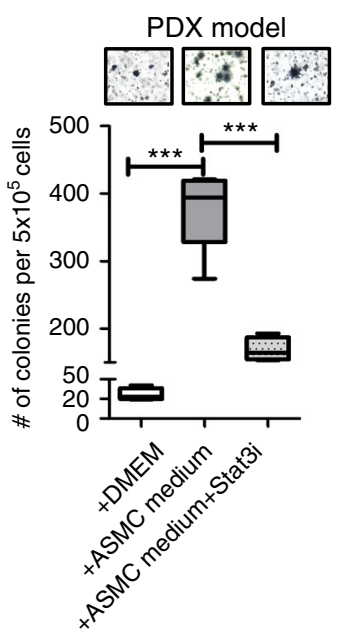

C
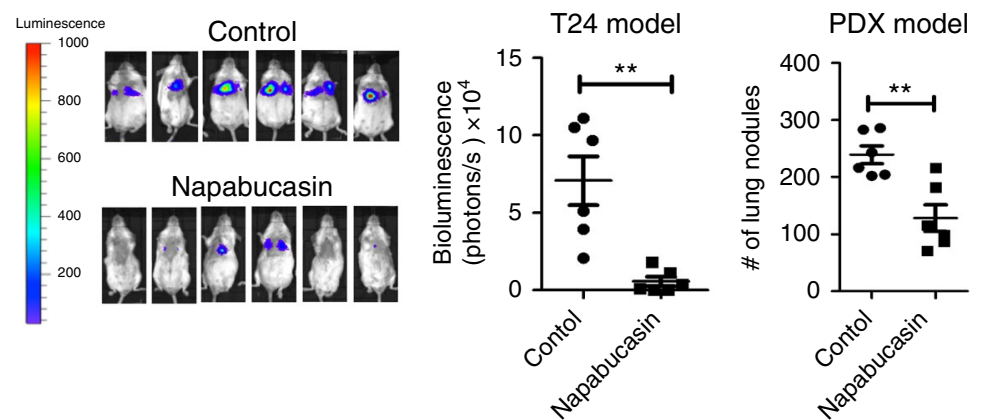

d
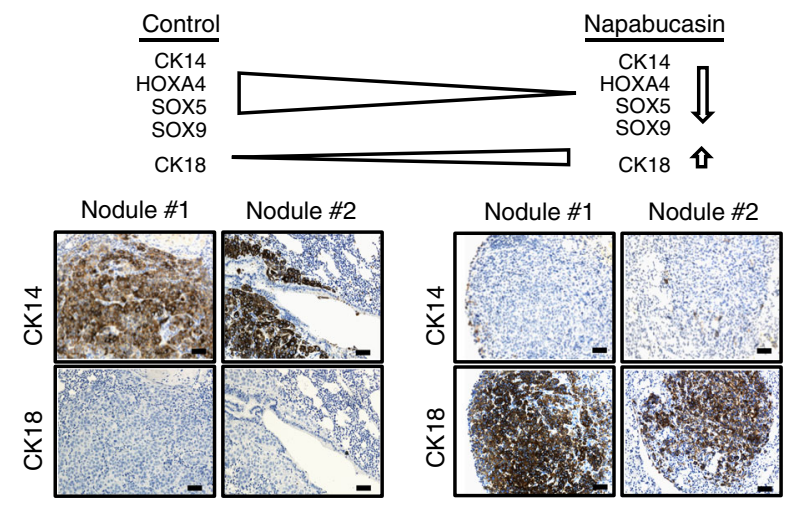

e
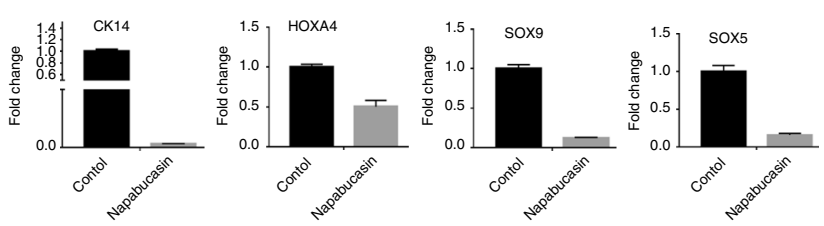

Fig. 7 Stat3 as a target to abrogate lung colonization. a, b Colony forming efficiency of T24 and metastatic PDX model upon treatment with Napabucasin (Stat3 inhibitor, $1 \mu \mathrm{M}$ ) in ASMC-conditioned media. Full colonies were allowed to form for 14 days, before the administration of vehicle or Napabucasin for an additional 7-day period. Live colonies were stained with the MTT assay for quantifications. c Assessment of metastatic lung colonization in T24 and metastatic PDX models upon treatment with Napabucasin for six weeks. $1 \times 10^{6}$ cancer cells were injected via tail-vein. Napabucasin treatment (intraperitoneal injection, $20 \mathrm{mg} \mathrm{kg}^{-1}$ ) started $72 \mathrm{~h}$ post-injection (T24 model, $n=6$; PDX model, $n=6$ ). BLI signals were measured weekly. BLI images at the experimental endpoint (after 6 weeks treatment) with respective quantitative intensities are shown. $\mathbf{d}$ Immunohistochemical analysis of stem/ progenitor and differentiation markers (CK14 and CK18) in vehicle and Napabucasin treated lung sections from mice injected with T24 cancer cells via tailvein. $\mathbf{e}$ Gene expression analysis of stemness markers (CK14, SOX5, SOX9 and HOXA4) in lung sections of vehicle and Napabucasin-treated mice tail-vein injected with T24 cancer cells. Statistical analysis: a two-tailed, unpaired student's t-test. Error bar: mean \pm SEM. ${ }^{\star} p<0.05,{ }^{\star \star} p<0.01$

it is interesting to note that Stat3 is an important gene for maintaining stemness, and Napabucasin was independently shown to block stem cell activity of cancer cells through targeting Stat3 activity $^{41}$. In our models, Napabucasin treatment similarly reduced the stemness property of lung colonizing cancer cells, illustrated by a significant decrease in the expression of stemness genes, e.g. CK14, SOX5, SOX9, and HOXA4, and an increased expression of the differentiated cell marker, CK18 (Fig. 7d, e). Collectively, these results revealed Stat 3 as an important survival signal to maintain lung colonization.

\section{Discussion}

The tumor microenvironment is emerging as an important component that contributes to tumor progression and metastasis. While it is recognized that many cell types, including stromal cells, immune cells, and vasculatures constitute the tumor microenvironment ${ }^{43-45}$, our study here focused on investigating the roles of collagens and their crosstalk with cancer cells (Fig. 8). Collagens were previously thought to be passive scaffolds within the ECM that prevents cancer cells from early invasion into their surroundings. Emerging studies have revealed collagens as functional ligands that crosstalk with collagen receptors on neighboring cancer cells ${ }^{46}$; however, this crosstalk remains an unexplored territory for bladder carcinomas. Our current study reports collagen I expression within human primary bladder tumors positively correlates with increasing tumor staging-an independent prognostic marker and clinical parameter measuring the extent of invasion into muscle and perivesical tissues. Functionally, while exogenous collagen I consistently induced cell migration in monolayer cell cultures, its effect in a $3 \mathrm{D}$ culture was significantly more pronounced, provoking both unique sprouting and invasive morphologies of bladder cancer cells. Our findings are well supported by studies from other cancer types, 

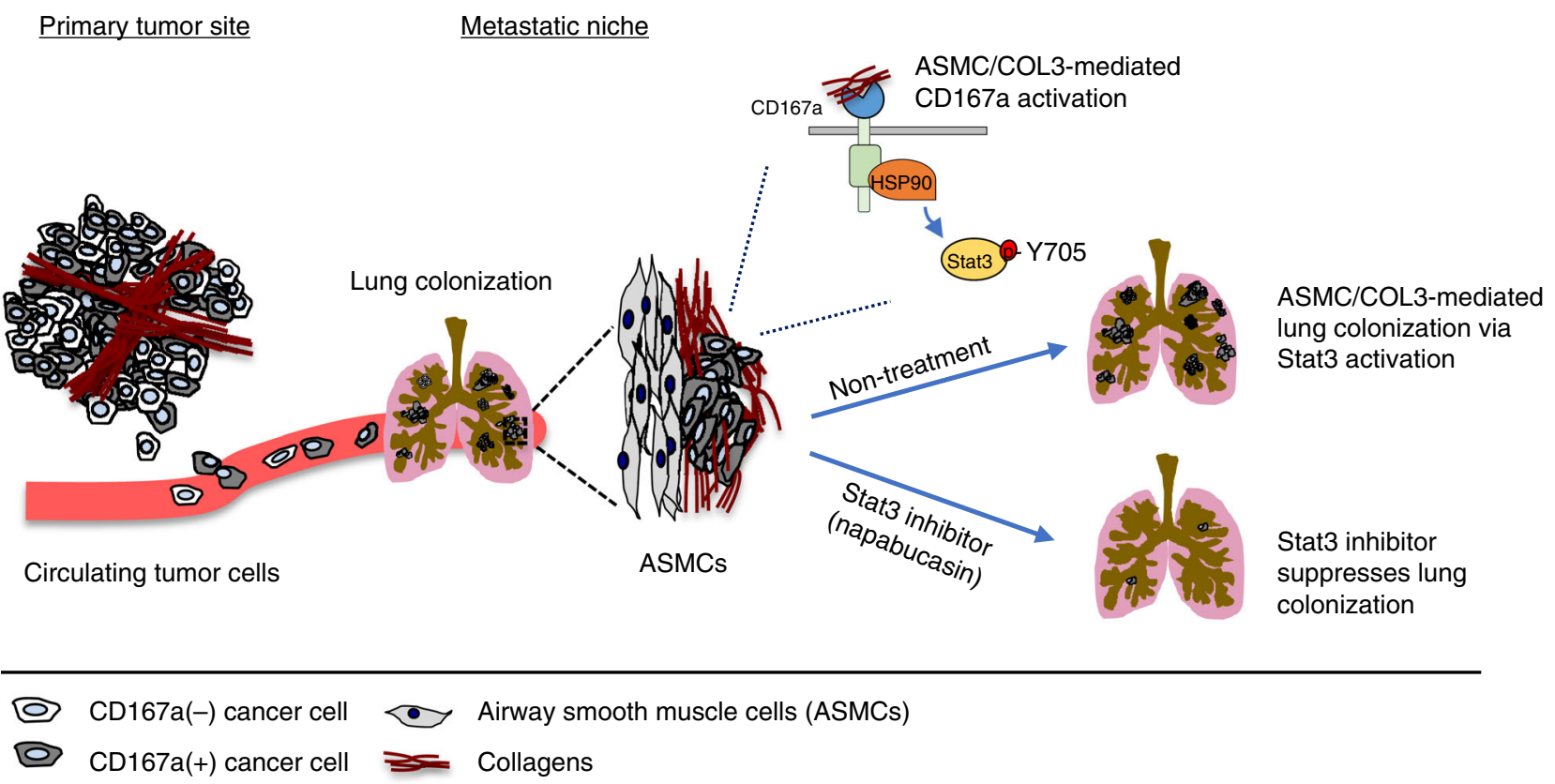

Fig. 8 Schematic diagram illustrating different collagens in the primary tumor and metastatic microenvironment and their regulation of the metastatic cascade. Airway smooth muscle cells are a collagen III rich niche for metastatic colonization of bladder cancer cells expressing CD167a, and Stat3 is a downstream target for abrogating these collagen III/CD167a-driven metastatic foci

demonstrating that collagen-CD167a signaling contributes to the invasive properties of cancer cells at the primary tumor site ${ }^{47}$.

While metastasis is a multi-step process, most studies linking collagen signaling to metastasis focuses on its roles during the initial steps of local invasion ${ }^{15-17}$. Data supporting a physical lung niche or microenvironment that facilitates metastatic colonization is not as well developed. While the thoughtprovoking premetastatic niche in lung was under extensive investigation $^{48,49}$, to our knowledge, the pre-existence of a collagen niche that supports metastatic tumor colonization in the lung has not been explored. Our results guided us into this new territory, by unraveling a remarkable pathologic phenotype of CD167a-expressing bladder cancer cells and their metastatic colonization to airway smooth muscle cells (ASMCs) (Fig. 8), representing an original finding not reported elsewhere. Initial search of literatures within the cancer research field did not provide us with any clues foremost ASMCs as a potential niche for metastatic colonization. By expanding our search to other research topics, we uncovered that airway remodeling by ASMCs is pertinent to asthma models ${ }^{29}$. Increased ECM deposition by ASMCs is a mechanism associated with ASM hyperplasia and hypertrophy, contributing to various clinical symptoms of asthma, including airway obstruction, shortness of breath, and chest pain or tightness ${ }^{30}$. These symptoms are often associated in advanced stage metastatic patients with heavy lung metastatic burden (Canadian Cancer Society http://www.cancer.ca/), highlighting the clinical relevance of our results. Further, ASMCs are reported to deposit collagens as the major ECM components, and collagen III was reported as an abundantly expressed collagen type within lung tissues ${ }^{31,32}$. We therefore investigated collagen III deposition as a probable mechanism favoring the colonization of CD167a-expressing metastatic foci to ASMCs in lung; since collagen III is an alternative ligand for CD167a $\mathrm{a}^{33,34}$. The role of collagen III in facilitating lung colonization was further reinforced by its capacity to activate the survival factor-Stat3. Pharmaceutical inhibition of Stat3 by Napabucasin-a drug already in Phase II/III clinical trials-significantly abrogated the capacity of CD167a-expressing cancer cells to form lung metastases. Others' and our previous studies independently revealed Stat3 expression as a hallmark of bladder cancer patients with the basal molecular subtype ${ }^{50,51}$. These findings present the rationale to evaluate anti-Stat3 drugs in future clinical trials, selecting metastatic bladder cancer patients with basal phenotype as the target population. This is clinically significant, since bladder cancer is the second most common urologic malignancy affecting men (causes 11,510 death) after prostate cancer (27,540 death; 2015 data from American Cancer Society). And current clinical prospects for metastatic bladder cancer patients are devastating, with a $6-8 \% 5$-year survival and limited treatment options ${ }^{11-13}$. While the newly FDA-approved immune checkpoint therapies created initial excitements for localized ${ }^{52}$ and invasive bladder cancers $^{43,53}$, they recently missed the expected endpoints in a Phase III clinical trial. This is interestingly and timely, since a recent study elegantly posed DDR2 (a family member of CD167a/DDR1) as a valid target to complement with anti-PD-1 immunotherapy ${ }^{54}$. Moreover, our data here also highlights the importance of organ-site specific research, a recent report using breast cancer models indicated an alternative noncanonical mechanism ${ }^{36}$, our data in bladder carcinomas implicated the canonical CD167a/DDR1 pathway for activating Stat3. Using proteomic discovery approach, we discovered HSP90 as an interacting protein with CD167a in our bladder cancer model. Blockade of CD167a kinase activity not only disrupted its interaction with HSP90, but also reduced Stat3 phosphorylation $^{55-57}$-revealing HSP90 as part of the canonical CD167a/ DDR1 signaling. Since HSP90 is a molecular chaperone that regulates many client proteins, this poses another interesting future research direction.

Additionally, our results here provide important insights to explain why the collagen receptor downstream signaling might be a good therapeutic target for the management of distant metastases $^{36,58}$. Metastatic bladder cancers are known to be unresponsive to cisplatin-based chemotherapy ${ }^{11-13}$, which could emerge from chemo-resistant clones within the primary tumors. Our recent report revealed collagen I deposition at the primary tumor as a hallmark of chemorefractory bladder cancer ${ }^{59,60}$, 
implicating a possible mechanism linking chemoresistance to metastatic progression. While it is somewhat intriguing that CD167a did not play a major role in other steps of the metastatic process, e.g. intravasation into circulation and extravasation from the circulation, our current study underscores the importance to carefully dissect each step of the metastatic cascade during mechanistic investigations. Together, our findings established a paradigm that CD167a-expressing cancer cells interacts with collagen I at the primary tumors to facilitate local invasion, and colonizes at collagen III-rich airway smooth muscle cells in lung (Fig. 8). While we recognize that other collagens are also expressed within these sites, our conception and recognition of the collagen microenvironments at the local and metastatic niche is timely. Recent exome sequencing of matched primary tumors and distal metastases from the same urothelial carcinoma patients remarkably revealed that they clonally evolved in parallel, resulting in tumor clones branching into distinct gene mutation patterns ${ }^{61}$. While no explanations were provided in that study, it seems plausible that distinct microenvironments at the local and metastatic sites likely contribute to such parallel progression of primary and metastatic tumor cells. Our data here provides insights to further investigate tumor-ECM interaction in the clonal evolution of primary and metastatic tumor cells in future studies. Our findings also revealed a generalized importance to convey ECMs and possibly other factors secreted by this ASMC metastatic niche to a broader audience for evaluation in other cancer types amid future studies.

\section{Methods}

Cell culture. The human bladder cancer cell line T24 was purchased from ATCC (HTB4). The highly metastatic T24 lung subline (LungMet line) was established from parental T24 cancer cells by serially re-injected ( 5 times) through tail-vein. Both cell lines were maintained in DMEM medium supplemented with $10 \%$ fetal bovine serum containing $100 \mu \mathrm{g} \mathrm{ml}^{-1}$ of streptomycin and $100 \mathrm{Units} / \mathrm{ml}$ of penicillin. Normal human primary airway smooth muscle cell (ASMC) line was purchased from ATCC $\left(\right.$ ATCC $^{\circledR}$ PCS-130-010 ${ }^{\text {Dit }}$ ) and cultured in vascular cell basal medium (ATCC ${ }^{\circledR}$ PCS$100-030^{\text {tw }}$ ) supplemented with vascular smooth muscle cell growth kit (ATCC ${ }^{\circledast}$ PCS$100-042^{\mathrm{TM}}$ ). All cells were free of mycoplasma contamination by PCR examination and incubated at $37^{\circ} \mathrm{C}$ in a humidified atmosphere containing $5 \% \mathrm{CO}_{2}$. To monitor cancer cell growth, CTCs and lung metastasis in vivo, T24 cells were labeled with a dual-fusion reporter vector expressing mCherry and click-beetle luciferase (mCherryCBLuc-T24 cells). For CD167a overexpression experiments, pHAGE-V5 or pHAGEV5-CD167a plasmids were used. CD167a/DDR1 contains five isoforms (a, b, c, d, and e), $b$ isoform of CD167a/DDR1 is the most abundant isoform and was used in the current study. These plasmids were packaged in 293T cells by co-transfection with compatible packaging plasmids. Culture supernatants containing the viral particles were collected $48 \mathrm{~h}$ post transfection and concentrated via high-speed centrifugation. mCherry-CBLuc-T24 cells were transduced with lentivirus-containing supernatant in the presence of $10 \mu \mathrm{g} \mathrm{ml}^{-1}$ polybrene for $24 \mathrm{~h}$ and replaced with selection medium containing $5 \mu \mathrm{g} \mathrm{ml}^{-1}$ puromycin for two weeks. The bladder PDX model was created by our laboratory from a pathologic T2 bladder cancer patient under the Institutional Animal Care and Use Committee (IACUC) protocol number H-25099. This human PDX tissue was ectopically embedded into immunocompromised mice, followed by an engraftment period of approximately two months, lung metastases were consistently observed. To culture the human bladder PDX line, DMEM/F12 medium supplemented with $10 \%$ fetal bovine serum containing $5 \mathrm{ml}$ GlutaMAX ${ }^{\mathrm{m}}$ solution, $20 \mathrm{ng} / \mathrm{ml}$ EGF, $10 \mathrm{mM}$ HEPES, 1X Insulin-Transferrin-Selenium (ITS-G), Gentamicin and $1.5 \mathrm{~g} / \mathrm{L} \mathrm{D}$-glucose was used. For the ASMC-conditioned medium experiments, vascular cell basal medium was removed from ASM cells, washed with PBS twice and subsequently cultured in DMEM medium overnight $(16 \mathrm{~h})$. The next day, ASMC-conditioned medium was transferred to T24 cancer cells or human bladder PDX line for another $24 \mathrm{~h}$; cells lysates were then harvested for subsequent analysis.

The clinical relevance of Collagen I gene expression in bladder cancer patients. The correlation between collagen I (COL1A1 and COL1A2) gene expression and the invasive properties (or clinical staging) of human bladder cancer specimens was evaluated by using three independent cohorts of bladder cancer patient samples [kim, $n=165^{20}$, Riester, $n=93^{21}$, and TCGA, $n=376^{22}$ ].

Antibodies and reagents. The following primary antibodies and dilutions were used: anti-CD167a at 1:1000 (WB) and 1:100 (IHC and IF) (Cell Signaling Technology, \#5583), anti-Stat3 at 1:1000 (WB) and 1:100 (IHC) (Cell Signaling Technology, \#9139), anti-phospho-Y705 Stat3 at 1:1000 (WB) (Cell Signaling
Technology, \#4113), anti-DNA-PKcs (G-4) at 1:1000 (Santa Cruz Biotechnology Inc., sc-5282); anti-GAPDH at 1:1000 (Santa Cruz Biotechnology Inc., sc-32233); anti-HSP90 $\alpha / \beta$ (F-8) at 1:1000 (Santa Cruz Biotechnology Inc., sc-13119); antiCollagen I at 1:100 (IHC) (Abcam ab34710), anti-Collagen III at 1:100 (IHC) (Abcam, ab7778), anti-mCherry at 1:100 (IHC) (Abcam ab167453) and anti- $\alpha$ SMA at 1:400 (IF) (SIGMA, \#A2547). Secondary antibodies were purchased from the following sources: for immunohistochemistry, anti-mouse and anti-rabbit horseradish peroxidase (HRP)-linked secondary antibodies were from Dako. For western blot analysis, anti-rabbit and anti-mouse HRP-conjugated secondary antibodies were from Cell signaling. For immunofluorescence analysis, goat antirabbit Alexa Fluor 488 (Z25302) and goat anti-mouse Alexa Fluor 594 (Z25207) were from Invitrogen. CD167a inhibitor, DDR1-IN1 was purchased from Selleckchem (S7498); Stat3 inhibitor, Napabucasin was purchased from Selleckchem (S7977); HSP90 inhibitor, Geldanamycin was purchased from Selleckchem (S2731) Protein agarose A/G beads for immunoprecipitation assays were purchased from Santa Cruz Biotechnology Inc., sc-2003. For collagen treatment, Collagen I was from purchased Corning (\#354236) and Collagen III was from Advanced BioMatrix (\#5021).

\section{Mass spectrometry-based immunoprecipitation (IP-MS) proteomics analysis} To identify DDR1/CD167a interacting proteins, cell lysates were collected from CD167a-overexpression T24 cells and using protein agarose A/G beads with antiCD167a antibody to perform immunoprecipitation assay. After $4 \mathrm{~h}$ incubation at $4{ }^{\circ} \mathrm{C}$, beads were washed with RIPA buffer for 4 times, and then IP samples were subjected to mass spectrometry analysis. Selected CD167a associated gene protein products (GPs) relative amounts from affinity purified samples was calculated using iBAQ algorithm by in-housed data processing algorithm. Simply, iBAQ was calculated based on normalization of summed peptide intensity (area-undercureve) of precursor ion MS signal divided by the number of theoretically observable tryptic peptide of certain protein.

Co-immunoprecipitation assay. Cell lysates were collected from T24 cells and human bladder PDX cultures treated with either COL3 $\left(25 \mu \mathrm{g} \mathrm{ml}^{-1}\right)$ or ASMCconditioned media, in the presence or absence of DDR1-IN1 $(10 \mu \mathrm{M})$ or Geldanamycin $(5 \mu \mathrm{M})$ for $24 \mathrm{~h}$. Five hundred micrograms of protein lysate (per sample) was incubated with $3 \mu \mathrm{g}$ of anti-HSP90 antibody and $20 \mu \mathrm{l}$ of protein agarose A/G beads at $4^{\circ} \mathrm{C}$ for $4 \mathrm{~h}$. Afterwards, beads were washed with RIPA buffer for four times, and then IP samples were subjected to western blot analysis.

Western blot analysis. Cell pellets or xenograft tissues were lysed in RIPA buffer (EMD Millipore) with complete protease and phosphatase inhibitor cocktails (Thermo Scientific). Lysates were collected by centrifugation at $18407 \times g$ for $15 \mathrm{~min}$ at $4^{\circ} \mathrm{C}$, and protein concentrations were measured by BCA assay. Twentyfive micrograms of sample lysates were subjected to western blot analysis using $4-12 \%$ Tris-Glycine gel under reducing conditions. Proteins were transferred onto PVDF membranes and probed with primary antibodies, anti-CD167a, Stat3, phospho-Y705 Stat3, HSP90 $\alpha / \beta$, and GAPDH were used at 1:1,000 dilution for standard immunoblotting with appropriate secondary HRP-conjugated antibodies (1:10,000 dilution). The bands were visualized using the enhanced chemiluminescence (ECL) system. Uncropped gel images are available in the Source Data.

Mass spectrometer analysis of ASMC conditioned medium. Parallel reaction monitoring (PRM) was implemented to validate the amount of collagen III in ASMC conditioned medium. The conditioned medium from ASM cells were collected at $0 \mathrm{~h}$, as control (incubated with ASM cells for $30 \mathrm{sec}$ ) and $16 \mathrm{~h}$ (after incubated with ASM cells for $16 \mathrm{~h}$ ), and subsequently subjected to mass spectrometeric analyses. We utilized the PRM method using Orbitrap Fusion ${ }^{\text {Tx }}$ Tribrid $^{\text {TM }}$ mass spectrometer. Depends on unique peptide availability, three or four unique peptides for each target protein was selected for PRM analysis. Pre-selected precursor ions were scanned with a $10 \mathrm{~min}$ predicted elution window and isolated by quadrupole followed by collisioninduced dissociation MS2 analysis. For relative quantification, the raw spectrum file was crunched to.mgf format by PD1.4 and then imported to Skyline with raw data file. We validated each result by deleting non-identified spectrum and adjusting the AUC range. Finally, the sum of the area of the three to four strongest product ions for each precursor ion (each peptide) were used for the result.

\section{Reverse transcription-PCR (RT-PCR) and quantitative real-time RT-PCR}

Total RNA of airway smooth muscle cells and T24 cells were isolated and reversetranscribed using the Aurum Total RNA mini kit (Cat. \#732-6820, BIO-RAD) and Reverse Transcription Supermix kit (Cat. \#1708841, BIO-RAD), respectively. The cDNA products were used to determine the mRNA expression of type III collagen with the primers list below. Quantitative real-time RT-PCR was performed by iTaq Universal SYBR Green Supermix (BI0-RAD, Cat. \#172-5121) and Roche LightCycler96 machine. The abundance of mRNA of type III collagen was normalized to GAPDH abundance.

GAPDH: Forward:5' -TGCACCACCAACTGCTTAGC-3

Reverse:5' - GGCATGGACTGTGGTCATGAG-3'

Collagen III: Forward: 5'-TCTTTGAATCCTAGCCCATCTG-3

Reverse: 5'-TGTGACAAAAGCAGCCCCATAA-3 
For the examination of collagen I receptors mRNA expression upon collagen I treatment, T24 cells were treated with or without $25 \mu \mathrm{g} \mathrm{ml}-1$ collagen I for $24 \mathrm{~h}$. Control and collagen I-treated cells were harvested and subjected to Quantitative real-time PCR using the following primers:

CD167a: Forward:5' - CCTGGGGACACTATCCTCATC-3'

Reverse:5'- GGATTGGAGAGCAGCAACG-3'

DDR2: Forward:5'- GCTATATGCCGCTATCCTCTGG-3'

Reverse:5' - ACTCTGACCACTGACTGGAAG-3'

ITGA1: Forward:5' - ACGCTGCTGCGTATCATTCA-3'

Reverse: $5^{\prime}$ - CAAACATGTCTTCCACCGGG-3'

ITGB1: Forward:5'- GTAACCAACCGTAGCAAAGGA-3'

Reverse:5'- TCCCCTGATCTTAATCGCAAAAC-3'

ITGA11: Forward:5'- GTCACCCTGTCCAACGTGTC-3'

Reverse:5'- ACATCCCTGTGGTGTAGTAGG-3'

MRC2: Forward:5'- ACATCACCATGGGTGTCGTC-3'

Reverse:5'- GGTTCTCTGGAAGCGCTGAT-3'

To examine the stemness-associated genes expression in control and Napabucasin-treated lung nodules, formalin-fixed, paraffin-embedded (FFPE)

lung sections were cut, collected and used High Pure FFPET RNA Isolation Kit (Roche, REF06650775001) to extract RNA and using the following primers for Quantitative real-time PCR:

CK14: Forward:5' - CTCACAGCCACAGTGGACAA-3'

Reverse:5'- TCATGCGCAGGTTCAACTCT-3'

CK18: Forward:5' - TATCACACGACTGCAGCTGG-3'

Reverse:5' - TGGCAATCTGGGCTTGTAGG-3'

HOXA4: Forward:5' - ATAACGGAGGGGAGCCTAAG-3'

Reverse:5'- GCTCAGACAAACAGAGCGTG-3'

SOX5: Forward:5'- CAGCCAGAGTTAGCACAATAGG-3'

Reverse:5'- CTGTTGTTCCCGTCGGAGTT-3'

SOX9: Forward:5'- AGCGAACGCACATCAAGAC-3'

Reverse:5' - CTGTAGGCGATCTGTTGGGG-3'

Two-dimensional wound-healing/scratch assay. For in vitro migration studies, cells were plated in triplicate on 96-well Essen Image Lock plates (Essen

BioScience) at 40,000 cells per well. Cancer cells were pre-treated with $30 \mathrm{ng} / \mathrm{ml}$ Mitomycin C for $2 \mathrm{~h}$ to block cell proliferation. The Essen Wound Maker (Essen BioScience) was used to make the wounds in confluent cell culture monolayers. Cancer cells were then treated with either 25 or $50 \mu \mathrm{g} \mathrm{ml}^{-1}$ of collagen I (Invitrogen) after scratch. Wound closure was monitored by acquiring images every $1 \mathrm{~h}$ over a $24 \mathrm{~h}$ period with the Incucyte ZOOM. An integrated metric called relative wound density (RWD) was used to quantify effects on migration. The grey area indicates the "wound gap" (devoid of cells), while the orange area indicates the movement of cancer cells migrating toward the gap area.

Three-dimensional cell culture and invasion assay. To determine whether collagen I can alter cells growth behavior in a 3D cell culture condition, a total of 2000 T24 cancer cells were cultured in Matrigel matrix (growth-factor reduced) mixed with or without collagen $\mathrm{I}\left(0.25 \mathrm{mg} \mathrm{m}^{-1}\right)$ and subsequently plated onto 96-well plates. Cancer cells were allowed to grow into colonies for an additional 14 days. Concurrently, new culture medium was added every 2 days to avoid the matrigel matrix drying. To mimic the local cell invasion in vivo, 3D collagen type I invasion assays were performed, the top gel was embedded with cancer cells at high density, in the absence or presence of increasing collagen I concentrations, while the bottom gel constituted an empty matrix to allow cancer cells to invade through. The collagen gels mixed with Matrigel ( $n=3$ gels per condition, $60 \mu \mathrm{l}$ volume) were prepared at a final concentration of $2.5 \mathrm{mg} \mathrm{ml}^{-1}$, and placed in a 96 -well plate. The collagen gels were allowed to polymerize and equilibrate at $37^{\circ} \mathrm{C}$ and $5 \%$ $\mathrm{CO}_{2}$ for $30 \mathrm{~min}$. After equilibration, 60,000 cells per well were seeded onto the surface of the gel and subsequently treated with the indicated concentration of collagen I $\left(0,10\right.$, and $\left.25 \mu \mathrm{g} \mathrm{ml}^{-1}\right)$, and then allowed to invade 3D collagen gels for $72 \mathrm{~h}$. At the time of collection, culture medium was removed and collagen matrices containing invading cancer cells were fixed in 4\% PFA for $20 \mathrm{~min}$. Matrices were stained with $0.1 \%$ toluidine blue in $30 \%$ methanol for $30 \mathrm{~min}$ prior to washing with water.

Immunohistochemistry (IHC) and immunofluorescence (IF) analysis. Tumor and lung tissues were collected from mice, and embedded in paraffin after formalin fixation at room temperature. For IHC analysis, tissue sections were heated at $65^{\circ} \mathrm{C}$ for 60-90 min, deparaffinized in xylene, rehydrated in an ethanol gradient, submerged in citrate buffer ( $\mathrm{pH} \mathrm{6.0)}$ and heated for $25 \mathrm{~min}$ to unmask antigens. Following a $3 \% \mathrm{H}_{2} \mathrm{O}_{2}$ treatment for $10 \mathrm{~min}$ to block endogenous peroxidase activity, serial sections were incubated with primary antibodies for $90 \mathrm{~min}$ at room temperature, washed with TBST buffer three times and then incubated with horseradish peroxidase (HRP)-conjugated secondary antibodies for $60 \mathrm{~min}$ at room temperature. Nuclei were counterstained with hematoxylin. For the IF analysis, the staining protocol was similar to IHC, after 90 min primary antibodies incubation, the tissue slides were washed with TBST buffer three times and then incubated with fluorescence-conjugated secondary antibodies (goat anti-rabbit Alexa Fluor 488 and goat anti-mouse Alexa Fluor 594) for $60 \mathrm{~min}$ at room temperature. Nuclei were counterstained with DAPI.

In vitro soft agar colony formation assay. The bottom of a six-well plate was coated with $1.5 \mathrm{~mL}$ of $0.7 \%$ low melting agarose, which was then covered with $1.5 \mathrm{~mL}$ of $0.35 \%$ agarose containing either 10,000 vector-control or CD167aoverexpressing mCherry-CBLuc T24 cells, or $5 \times 10^{5}$ of the human bladder PDX cells. To determine the effect of Collagen III on colony formation, $0.35 \%$ agarose gel was mixed with $50 \mu \mathrm{g} \mathrm{ml}^{-1}$ Collagen III. Regularly, the six-well plates were incubated for 14 days at $37^{\circ} \mathrm{C}$ with $5 \% \mathrm{CO}_{2}$. DMEM medium was added every other day to the upper agar layer to prevent desiccation. To examine the effect of ASM cells on the colony formation of cancer cells, DMEM was replaced with ASMC-conditioned medium to add to the cells. For Stat 3 inhibition experiments, $1 \mu \mathrm{M}$ Napabucasin or vehicle was added to the cells at day 14 of the assay and incubated for an additional 7 days. Live colonies were stained with the MTT reagent (3-(4,5-dimethylthiazol-2-yl)-2,5-diphenyltetrazolium bromide) for $2 \mathrm{~h}$ and quantified at the end point.

Xenograft tumor growth. A total of $1 \times 10^{6}$ vector-control and CD167a overexpression mCherry-CBLuc T24 cells were injected into the 6 to 8 week-old $\mathrm{Rag}^{-1-}$; IL-2R $\mathrm{C}^{-/-}$immunocompromised mice. All animals were treated in accordance with IUCAC guidelines. The body weight of each mice and their corresponding tumor size were measured to observe how tumor progression impacted the health status of each mouse. Tumor diameters were measured at regular intervals with a caliper, and the tumor volume in $\mathrm{mm}^{3}$ was calculated weekly by the formula: volume $=$ length $\times$ width $\times$ height. The tumors were isolated and snap-frozen in liquid nitrogen for western blot analysis or fixed with $3.7 \%$ formalin for immunohistochemical analysis at the end of experimental time point.

Flow cytometry analysis. To examine the frequency of CTC, equal volume $(100 \mu \mathrm{L})$ of whole peripheral blood was collected simultaneously from vectorcontrol and CD167a-overexpressing mCherry-CBLuc mice. Red blood cells were lysed by the addition Ammonium Chloride solution (ACK), washed and then subjected to flow cytometry for single and clustered CTCs analysis. CTCs detection was based on fluorescent protein (i.e., mCherry) expression. Distinction between single and clustered CTCs was based on the side scattered (SSC)-area vs SSCwidth plot.

Tail-vein injection model to evaluate extravasation and lung colonization. To select for a highly lung metastatic T24 subline, $1 \times 10^{6}$ mCherry-CBLuc-T24 cells were injected into mice by tail-vein. Tumor growth was monitored by bioluminescent signal using in vivo IVIS imaging. Mice carrying heavy lung metastatic burden were sacrificed and lung tissues containing metastatic foci were enzymatically dissociated for re-injected into mice via the tail-vein. This experimental selection was repeated five consecutive times.

To evaluate the role of CD167a during extravasation and/or colonization, $1 \times 10^{6}$ vector-control or CD167a overexpression mCherry-CBLuc T24 cancer cells were injected into mice via tail-vein and extravasation was assessed measuring the bioluminescence signal intensity at $72 \mathrm{~h}$ post injection. Lung colonization was evaluated by monitoring the bioluminescence signal in the lung weekly for a total of 5 weeks. To examine the in vivo effect of the Stat 3 inhibitor, Napabucasin, in modulating metastatic lung colonization, mice were treated with Napabucasin (20 $\mathrm{mg} \mathrm{kg}^{-1}$, intraperitoneal injection) or vehicle control at $72 \mathrm{~h}$ post tail-vein injection of cancer cells. Mice were treated daily for 3 weeks with Napabucasin and then every other day for another 3 weeks, for a total of 6-weeks treatment.

Statistics. Student's two sample $t$-test was used to determine if there was statistical difference between the means of two groups (e.g. control and experimental groups). Analysis of Variance (ANOVA) was used for comparing three or more group means. $P$-values were two-sided and $p$-value $<0.05$ was considered statistically significant. Quantified data shown represent at least three independent experiments. Data were represented as mean \pm SEM.

Study approval. Animal experiments were conducted according to a protocol reviewed and approved by the IACUC of Baylor College of Medicine.

Reporting Summary. Further information on research design is available in the Nature Research Reporting Summary linked to this article.

\section{Data availability}

The data that support the findings of this study are available from the corresponding author upon reasonable request.

Received: 18 January 2018 Accepted: 27 March 2019

Published online: 13 May 2019 


\section{References}

1. Brabletz, T., Lyden, D., Steeg, P. S. \& Werb, Z. Roadblocks to translational advances on metastasis research. Nat. Med. https://doi.org/10.1038/nm.3327 (2013).

2. Wan, L., Pantel, K. \& Kang, Y. Tumor metastasis: moving new biological insights into the clinic. Nat. Med. https://doi.org/10.1038/nm.3391 (2013)

3. Massagué, J. \& Obenauf, A. C. Metastatic colonization by circulating tumour cells. Nature. https://doi.org/10.1038/nature17038 (2016).

4. Lambert, A. W., Pattabiraman, D. R. \& Weinberg, R. A. Emerging biological principles of metastasis. Cell. https://doi.org/10.1016/j. cell.2016.11.037 (2017).

5. Celià-terrassa, T. \& Kang, Y. Distinctive properties of metastasis- initiating cells. Genes Dev. https://doi.org/10.1101/gad.277681.116.892 (2016).

6. Van Der Weyden, L. et al. Genome-wide in vivo screen identifies novel host regulators of metastatic colonization. Nature. https://doi.org/10.1038/ nature20792 (2017).

7. Stenzl, A. et al. Treatment of muscle-invasive and metastatic bladder cancer: update of the EAU guidelines. Eur. Urol. https://doi.org/10.1016/j. eururo.2011.03.023. (2011).

8. Calabro, F. \& Sternberg, C. N. Metastatic bladder cancer: anything new? Curr. Opin. Support. Palliat. Care. https://doi.org/10.1097/SPC.0b013e3283552d19 (2012).

9. Carballido, E. M. \& Rosenberg, J. E. Optimal treatment for metastatic bladder cancer. Curr. Oncol. Rep. https://doi.org/10.1007/s11912-014-0404-2 (2014).

10. Collazo-Lorduy, A. \& Galsky, M. D. Systemic therapy for metastatic bladder cancer in 2016 and beyond. Future Oncol. https://doi.org/10.2217/fon-20150020 (2016).

11. Chaffer, C. L. et al. Mesenchymal-to-epithelial transition facilitates bladder cancer metastasis: role of fibroblast growth factor receptor-2. Cancer Res. https://doi.org/10.1158/0008-5472.CAN-06-2044 (2006).

12. Overdevest, J. B. et al. CD24 offers a therapeutic target for control of bladder cancer metastasis based on a requirement for lung colonization. Cancer Res. https://doi.org/10.1158/0008-5472.CAN-11-0519 (2011).

13. Said, N., Smith, S., Sanchez-Carbayo, M. \& Theodorescu, D. Tumor endothelin-1 enhances metastatic colonization of the lung in mouse xenograft models of bladder cancer. J. Clin. Invest. https://doi.org/10.1172/JCI42912 (2011).

14. Egeblad, M., Nakasone, E. S. \& Werb, Z. Tumors as organs: complex tissues that interface with the entire organism. Dev. Cell. https://doi.org/10.1016/j. devcel.2010.05.012. (2010).

15. Egeblad, M., Rasch, M. G. \& Weaver, V. M. Dynamic interplay between the collagen scaffold and tumor evolution. Curr. Opin. Cell Biol. https://doi.org/ 10.1016/j.ceb.2010.08.015. (2010).

16. Lu, P., Weaver, V. M. \& Werb, Z. The extracellular matrix: a dynamic niche in cancer progression. J. Cell Biol. https://doi.org/10.1083/jcb.201102147 (2012).

17. Pickup, M. W., Mouw, J. K. \& Weaver, V. M. The extracellular matrix modulates the hallmarks of cancer. EMBO Rep. https://doi.org/10.15252/ embr.201439246. (2014).

18. Psaila, B. \& Lyden, D. The metastatic niche: adapting the foreign soil Nat. Rev. Cancer. https://doi.org/10.1038/nrc2621 (2009).

19. Knowles, M. A. \& Hurst, C. D. Molecular biology of bladder cancer: new insights into pathogenesis and clinical diversity. Nat. Rev. Cancer. https://doi. org/10.1038/nrc3817 (2015).

20. Kim, W. J. et al. Predictive value of progression-related gene classifier in primary non-muscle invasive bladder cancer. Mol. Cancer. https://doi.org/ 10.1186/1476-4598-9-3 (2010).

21. Riester, M. et al. Integrative analysis of 1q23.3 copy-number gain in metastatic urothelial carcinoma. Clin. Cancer Res. https://doi.org/10.1158/1078-0432. CCR-13-0759 (2014).

22. Robertson, A. G. et al. Comprehensive molecular characterization of muscleinvasive bladder cancer. Cell. https://doi.org/10.1016/j.cell.2017.09.007. (2017).

23. Fang, M., Yuan, J., Peng, C. \& Li, Y. Collagen as a double-edged sword in tumor progression. Tumor Biol. https://doi.org/10.1007/s13277-013-1511-7 (2014).

24. Seraj, M. J., Harding, M. a, Gildea, J. J., Welch, D. R. \& Theodorescu, D. The relationship of BRMS1 and RhoGDI2 gene expression to metastatic potential in lineage related human bladder cancer cell lines. Clin. Exp. Metastasis 18:519-25 (2000)

25. Liang, C.-C., Park, A. Y. \& Guan, J.-L. In vitro scratch assay: a convenient and inexpensive method for analysis of cell migration in vitro. Nat. Protoc. https://doi.org/10.1038/nprot.2007.30. (2007).

26. Bayless, K. J., Kwak, H. Il \& Su, S. C. Investigating endothelial invasion and sprouting behavior in three-dimensional collagen matrices. Nat. Protoc. https://doi.org/10.1038/nprot.2009.221. (2009).

27. Aceto, N. et al. Circulating tumor cell clusters are oligoclonal precursors of breast cancer metastasis. Cell. https://doi.org/10.1016/j.cell.2014.07.013 (2014).

28. Elkin, M. \& Vlodavsky, I. Tail vein assay of cancer metastasis. Curr. Protoc. Cell Biol. https://doi.org/10.1002/0471143030.cb1902s12 (2001). in.
29. Parameswaran, K. et al. Role of extracellular matrix and its regulators in human airway smooth muscle biology. Cell Biochem. Biophys. https://doi.org/ 10.1385/CBB:44:1:139 (2006).

30. Noble, P. B. et al. Airway smooth muscle in asthma: Linking contraction and mechanotransduction to disease pathogenesis and remodelling. Pulm. Pharmacol. Ther. https://doi.org/10.1016/j.pupt.2014.07.005. (2014).

31. Bradley, K. H., McConnell, S. D. \& Crystal, R. G. Lung collagen composition and synthesis. Characterization and changes with age. J. Biol. Chem. 10;249:2674-83 (1974)

32. Bradley, K., McConnell-Breul, S. \& Crystal, R. G. Lung collagen heterogeneity. Proc. Natl Acad. Sci. USA https://doi.org/10.1073/pnas.71.7.2828 (1974).

33. $\mathrm{Xu}, \mathrm{H}$. et al. Collagen binding specificity of the discoidin domain receptors: Binding sites on collagens II and III and molecular determinants for collagen IV recognition by DDR1. Matrix Biol. https://doi.org/10.1016/j. matbio.2010.10.004 (2011)

34. Vogel, W., Gish, G. D., Alves, F. \& Pawson, T. The discoidin domain receptor tyrosine kinases are activated by collagen. Mol. Cell. https://doi.org/10.1016/ S1097-2765(00)80003-9 (1997).

35. Kim, H.-G. et al. Discovery of a potent and selective DDR1 receptor tyrosine kinase inhibitor. ACS Chem. Biol. https://doi.org/10.1021/cb400430t (2013).

36. Gao, $\mathrm{H}$. et al. Multi-organ site metastatic reactivation mediated by noncanonical discoidin domain receptor 1 signaling. Cell. https://doi.org/10.1016/ j.cell.2016.06.009 (2016)

37. Jung, S. Y. et al. An anatomically resolved mouse brain proteome reveals Parkinson disease-relevant pathways. Mol. Cell. Proteom. https://doi.org/ 10.1074/mcp.M116.061440 (2017).

38. Schopf, F. H., Biebl, M. M. \& Buchner, J. The HSP90 chaperone machinery. Nat. Rev. Mol. Cell Biol. https://doi.org/10.1038/nrm.2017.20 (2017).

39. Prinsloo, E., Kramer, A. H., Edkins, A. L. \& Blatch, G. L. STAT3 interacts directly with Hsp90. IUBMB Life. https://doi.org/10.1002/iub.607 (2012).

40. Bocchini, C. E., Kasembeli, M. M., Roh, S.-H. \& Tweardy, D. J. Contribution of chaperones to STAT pathway signaling. JAK-STAT. https://doi.org/10.4161/ 21623988.2014.970459 (2014).

41. Hubbard, J. M. \& Grothey, A. Napabucasin: an update on the first-in-class cancer stemness inhibitor. Drugs. https://doi.org/10.1007/s40265-017-0759-4 (2017).

42. Jonker, D. J. et al. Napabucasin versus placebo in refractory advanced colorectal cancer: a randomised phase 3 trial. Lancet Gastroenterol. Hepatol. https://doi.org/10.1016/S2468-1253(18)30009-8 (2018).

43. Shin, K. et al. Hedgehog signaling restrains bladder cancer progression by eliciting stromal production of urothelial differentiation factors. Cancer Cell. https://doi.org/10.1016/j.ccell.2014.09.001 (2014).

44. Roberts, K. J., Kershner, A. M. \& Beachy, P. A. The stromal niche for epithelial stem cells: a template for regeneration and a brake on malignancy. Cancer Cell. https://doi.org/10.1016/j.ccell.2017.08.007 (2017).

45. Quail, D. F. \& Joyce, J. A. Microenvironmental regulation of tumor progression and metastasis. Nat. Med. https://doi.org/10.1038/nm.3394 (2013).

46. Kim, S. H., Turnbull, J. \& Guimond, S. Extracellular matrix and cell signalling: The dynamic cooperation of integrin, proteoglycan and growth factor receptor. J. Endocrinol.. https://doi.org/10.1530/JOE-10-0377 (2011).

47. Shintani, Y. et al. Collagen I-mediated up-regulation of $\mathrm{N}$-cadherin requires cooperative signals from integrins and discoidin domain receptor. J. Cell Biol. https://doi.org/10.1083/jcb.200708137 (2008).

48. Kaplan, R. N., Rafii, S. \& Lyden, D. Preparing the 'soil': The premetastatic niche. Cancer Res.. https://doi.org/10.1158/0008-5472.CAN-06-2407 (2006).

49. Prakash, Y. S. Emerging concepts in smooth muscle contributions to airway structure and function: implications for health and disease. Am. J. Physiol. Lung Cell. Mol. Physiol. https://doi.org/10.1152/ajplung.00370.2016 (2016).

50. Mo, Q. et al. Prognostic power of a tumor differentiation gene signature for bladder urothelial carcinomas. J. Natl. Cancer Inst. https://doi.org/10.1093/ jnci/djx243 (2018)

51. Eriksson, P. et al. Molecular subtypes of urothelial carcinoma are defined by specific gene regulatory systems. BMC Med. Genom.. https://doi.org/10.1186/ s12920-015-0101-5 (2015)

52. Rampias, T. et al. A new tumor suppressor role for the Notch pathway in bladder cancer. Nat. Med. https://doi.org/10.1038/nm.3678 (2014).

53. Hayashi, T. et al. Not all NOTCH is created equal: the oncogenic role of notch2 in bladder cancer and its implications for targeted therapy. Clin. Cancer Res. https://doi.org/10.1158/1078-0432.CCR-15-2360 (2016)

54. Tu, M. A. et al. Targeting DDR2 enhances tumor response to anti-PD-1 immunotherapy. Sci. Adv. 5, eaav2437 (2019).

55. Hosoi, T. et al. Key role of heat shock protein 90 in leptin-induced STAT3 activation and feeding regulation. Br. J. Pharmacol. https://doi.org/10.1111/ bph.13520 (2016).

56. Sato, N. et al. Involvement of heat-shock protein 90 in the interleukin-6mediated signaling pathway through STAT3. Biochem. Biophys. Res. Commun. https://doi.org/10.1016/S0006-291X(02)02941-8 (2003). 
57. Shah, M., Patel, K., Fried, V. A. \& Sehgal, P. B. Interactions of STAT3 with caveolin-1 and heat shock protein 90 in plasma membrane raft and cytosolic complexes: Preservation of cytokine signaling during fever. J. Biol. Chem. https://doi.org/10.1074/jbc.M205935200 (2002).

58. Valencia, K. et al. Inhibition of collagen receptor discoidin domain receptor-1 (DDR1) reduces cell survival, homing, and colonization in lung cancer bone metastasis. Clin. Cancer Res. https://doi.org/10.1158/1078-0432.CCR-11-1686 (2012).

59. Kurtova, A. V. et al. Blocking PGE2-induced tumour repopulation abrogates bladder cancer chemoresistance. Nature. https://doi.org/10.1038/nature14034 (2015).

60. Chan, K. S. Molecular Pathways: Targeting Cancer Stem Cells Awakened by Chemotherapy to Abrogate Tumor Repopulation. Clin. Cancer Res. 22, 802-806 (2016)

61. Faltas, B. M. et al. Clonal evolution of chemotherapy-resistant urothelial carcinoma. Nat. Genet. https://doi.org/10.1038/ng.3692 (2016).

\section{Acknowledgements}

This work was supported by NCI R01-CA175397 (KSC), CPRIT RP140252, V Foundation V Scholar Award (KSC), and in part by CPRIT Core Facility Support Awards: RP120348 and RP170002, CPRIT Core Facility Award: RP170005 and P30 Cancer Center support Grant: NCI-CA125123. We would like to thank the generosity of Dr. Y.H. Tsang and the late Dr. Kenneth Scott in providing the pHAGE-V5 vector, Dr. H. Kang in experimental assistance, and the technical assistance from Dr. Zhang, Ms. J. Liu and Dr. Nakada's groups during manuscript revision.

\section{Author contributions}

Y.C.L., A.V.K., J.X., F.N., and Z.T. performed the experiments and collected, analyzed, and discussed the data; Y.C.L. and K.S.C. managed and designed the study, conceived the experiments, and wrote the manuscript. F.N. and K.H. edited the manuscript. F.C., J.S., and Q.M. performed bioinformatics analysis, X.Z. and D.R. contributed to the discussion of the study. S.Y.J. and A.J. assisted the mass spectrometry proteomics analysis. M.C., C.L., and G.B. assisted experiments.

\section{Additional information}

Supplementary Information accompanies this paper at https://doi.org/10.1038/s41467019-09878-4.

Competing interests: The authors declare no competing interests.

Reprints and permission information is available online at http://npg.nature.com/ reprintsandpermissions/

Journal peer review information: Nature Communications thanks Jean Paul Thiery and other anonymous reviewer(s) for their contribution to the peer review of this work.

Publisher's note: Springer Nature remains neutral with regard to jurisdictional claims in published maps and institutional affiliations.

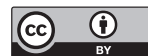

Open Access This article is licensed under a Creative Commons Attribution 4.0 International License, which permits use, sharing, adaptation, distribution and reproduction in any medium or format, as long as you give appropriate credit to the original author(s) and the source, provide a link to the Creative Commons license, and indicate if changes were made. The images or other third party material in this article are included in the article's Creative Commons license, unless indicated otherwise in a credit line to the material. If material is not included in the article's Creative Commons license and your intended use is not permitted by statutory regulation or exceeds the permitted use, you will need to obtain permission directly from the copyright holder. To view a copy of this license, visit http://creativecommons.org/ licenses/by/4.0/.

(C) The Author(s) 2019 